\title{
A GEOMETRIC APPROACH TO REGULAR PERTURBATION THEORY WITH AN APPLICATION TO HYDRODYNAMICS
}

\author{
CARMEN CHICONE
}

\begin{abstract}
The Lyapunov-Schmidt reduction technique is used to prove a persistence theorem for fixed points of a parameterized family of maps. This theorem is specialized to give a method for detecting the existence of persistent periodic solutions of perturbed systems of differential equations. In turn, this specialization is applied to prove the existence of many hyperbolic periodic solutions of a steady state solution of Euler's hydrodynamic partial differential equations. Incidentally, using recent results of S. Friedlander and M. M. Vishik, the existence of hyperbolic periodic orbits implies the steady state solutions of the Eulerian partial differential equation are hydrodynamically unstable. In addition, a class of the steady state solutions of Euler's equations are shown to exhibit chaos.
\end{abstract}

\section{INTRODUCTION}

The existence of solutions for many perturbation problems can be reduced to the question of the persistence of fixed points of a map of the type $(\xi, \epsilon) \mapsto$ $\mathscr{P}(\xi, \epsilon)$ where $\xi$ is a state variable and $\epsilon$ is a small parameter. When the unperturbed function $\xi \mapsto \mathscr{P}(\xi, 0)$ has a fixed point, $z$, we say $z$ persists if there is a function $\epsilon \mapsto \beta(\epsilon)$, defined in a neighborhood of $\epsilon=0$, such that $\beta(0)=z$ and $\mathscr{P}(\beta(\epsilon), \epsilon) \equiv \beta(\epsilon)$.

In case $z$ is a simple fixed point of the unperturbed map, the fact that $z$ persists is an immediate consequence of the Implicit Function Theorem. However, in many important applications, the Implicit Function Theorem does not apply because the fixed point set of the unperturbed function is a manifold of dimension exceeding zero. In the analysis of these applications, some method of reduction-reduction to the Implicit Function Theorem-can often be used to obtain the implicit solution of the state variable in terms of the perturbation parameter in order to prove persistence.

In a series of papers, [5], [6], [7], we have developed a reduction method for the persistence problem based on the classical Lyapunov-Schmidt reduction. Using some of the ideas from this theory, we state and prove here a general version of the reduction method that is applicable in the context of maps defined on smooth manifolds. As a corollary of this theorem, we obtain the conditions

Received by the editors April 8, 1994.

1991 Mathematics Subject Classification. Primary 58F14, 58F22, 58F30, 34C15, 34C25.

Key words and phrases. Lyapunov-Schmidt reduction, resonance, normal nondegeneracy, Euler equations, chaos.

This research was supported by the National Science Foundation under grant DMS-9303767. 
for persistence of periodic solutions of a family of differential equations in case the unperturbed system has an invariant manifold of periodic solutions. A reasonable theory for persistence using first order methods is possible in case this invariant manifold satisfies a verifiable property we call normal nondegeneracy. It turns out that this condition is satisfied if the invariant manifold is normally hyperbolic. However, as we will see, the theory has important applications in cases when the invariant manifold does not satisfy this stronger condition.

As an application of our theory, we will extend some recent results of $S$. Friedlander, A. Gilbert and M. Vishik [9] on the existence of periodic solutions of a certain steady state solution of Euler's hydrodynamic equations.

Recall that the equations of motion of an ideal fluid in a three dimensional bounded region are given by Bernoulli's form of Euler's equations as

$$
\frac{\partial V}{\partial t}=V \wedge \operatorname{curl} V+\operatorname{grad} \alpha, \quad \operatorname{div} V=0,
$$

where $V$ denotes the velocity of the fluid and $\alpha$ is a function determined by the fact that $\operatorname{div} V=0$ and the additional condition that $V$ is tangent to the boundary of the region. For the idealized case of steady flow on the three torus $\mathbb{T}^{3}$, the boundary in empty. Thus, we can take $\alpha=0$ and seek "curl parallel" [4] steady state solutions on $\mathbb{T}^{3}$, that is, solutions of $V \wedge \operatorname{curl} V=0$.

The vector field on $\mathbb{T}^{3}$ associated with the $\mathrm{ABC}$ system, namely,

$$
\begin{aligned}
& \dot{x}=A \sin z+C \cos y, \\
& \dot{y}=B \sin x+A \cos z, \\
& \dot{z}=C \sin y+B \cos x,
\end{aligned}
$$

where the real coordinates $x, y$ and $z$ are viewed modulo $2 \pi$, is curl parallel. This system was introduced by V. I. Arnold [1] and studied in [9] with regard to the hydrodynamic instability criterion of S. Friedlander and M. M. Vishik [10], [11]. In particular, their results show that the existence of a single hyperbolic periodic solution of an $A B C$ system implies the associated steady state solution of Euler's equation is hydrodynamically unstable.

The main result of [9] is the following perturbation theorem: Consider (1) with $A=1$ and $\epsilon:=B=C$. If $\epsilon>0$ is sufficiently small, then (1) has hyperbolic periodic solutions. We will give a new proof of this result and prove a more general result for the case when only one of the $\mathrm{ABC}$ parameters is small. In particular, we will prove the following perturbation theorem: Consider (1) with $A=1,0<B \leq 1$ and $\epsilon:=C$. If $N$ is a positive integer, then there is some $\epsilon>0$ such that (1) has at least $N$ hyperbolic periodic solutions. Actually, our results determine exactly. which resonances are excited (at first order) as well as the number and position of the persistent periodic solutions.

As an addendum to our results on the persistence of periodic solutions for the case of one small parameter, we also show that the unperturbed $A B C$ system on $\mathbb{T}^{3}$ has a homoclinic orbit that breaks after perturbation into transversally intersecting stable and unstable manifolds of a hyperbolic periodic orbit of saddle type. By the Smale-Birkoff Theorem, this implies the perturbed system exhibits chaos-there is an invariant Cantor set in a cross section of the flow on which the associated Poincare map is topologically conjugate to a full shift. Moreover, 
there are infinitely many hyperbolic periodic orbits of the perturbed flow passing through this invariant set.

In Section 2 the general persistence theorem based on the Lyapunov-Schmidt reduction is presented. In Section 3 we construct special coordinate systems called normal coordinates that are convenient for applications of the general theorem to dynamical systems. The specialization of the theory to perturbations of oscillators is completed in Section 4 while the application to ABC flows is carried out in Section 5.

\section{LYAPUNOV-SCHMIDT REDUCTION}

Let $\mathscr{Z}$ denote a smooth open submanifold of $\mathscr{Y}$ and $\mathscr{P}: \mathscr{Z} \times \mathbb{R} \rightarrow \mathscr{Y}$ a smooth function given by $(\xi, \epsilon) \mapsto \mathscr{P}(\xi, \epsilon)$. Also, define the associated unperturbed function $p: \mathscr{Z} \rightarrow \mathscr{Y}$ by $p(\xi):=\mathscr{P}(\xi, 0)$. We say a fixed point, $z \in \mathscr{X}$, of the unperturbed function persists provided there is a curve $\epsilon \mapsto \beta(\epsilon)$ in $\mathscr{Z}$, defined on some open interval of $\mathbb{R}$ containing $\epsilon=0$, such that $\beta(0)=$ $z$ and $\mathscr{P}(\beta(\epsilon), \epsilon) \equiv \beta(\epsilon)$. In this section, we propose a method to determine which fixed points of the unperturbed function persist.

As we will soon see, persistence is a local phenomenon. However, it is useful to formulate our results without reference to coordinate systems. Thus, in this section, we will use the language of manifolds and maps. In particular, if $\mathscr{Z}$ and $\mathscr{Z}$ are manifolds and $z \in \mathscr{Z}$, then we let $\mathrm{T}_{z} \mathscr{Z}$ denote the tangent space to $\mathscr{Z}$ at the point $z$ while $\mathrm{T} \mathscr{Z}$ denotes the tangent bundle of the manifold $\mathscr{Z}$. If, in addition, $\mathscr{Z} \subseteq \mathscr{X}$ is a submanifold of $\mathscr{X}$, we let $\mathrm{T}_{\mathscr{X}} \mathscr{X}$ denote the restriction of the tangent bundle of $\mathscr{X}$ to $\mathscr{Z}$. Also, we note that $\mathrm{T} \mathscr{Z}$ is naturally viewed as a subbundle of $\mathrm{T}_{\mathscr{Z}} \mathscr{X}$.

The derivative (also called the tangent map) of a smooth map $f: \mathscr{Z} \rightarrow \mathscr{X}$ is denoted $\mathrm{T} f: \mathrm{T} \mathscr{Z} \rightarrow \mathrm{T} \mathscr{Z}$. Recall that, abstractly, a tangent vector in $\mathrm{T}_{z} \mathscr{Z}$ is an equivalence class of curves. If fact, if $\mathscr{Z}$ has dimension $k$, two smooth curves $s \mapsto \gamma(s)$ and $s \mapsto \sigma(s)$ are equivalent if $\gamma(0)=\sigma(0)=z$ and if there is a local coordinate $\phi: \mathbb{R}^{k} \rightarrow \mathscr{Z}$ such that

$$
\left.\frac{d}{d s} \phi^{-1}(\gamma(s))\right|_{s=0}=\left.\frac{d}{d s} \phi^{-1}(\sigma(s))\right|_{s=0} .
$$

If we denote the equivalence class of a curve $\gamma$ by $[\gamma]$, the derivative $\mathrm{T} f$ is defined by

$$
\mathrm{T} f[\gamma]=[f \circ \gamma] \text {. }
$$

The tangent space $\mathrm{T}_{z} \mathscr{Z}$ is a $k$ dimensional vector space; it inherits the linear structure of $\mathbb{R}^{k}$. With this definition, we observe that the restriction of $\mathrm{T} f$ to $\mathrm{T}_{z} \mathscr{Z}$, that we denote by $\mathrm{T}_{z} f$, is a linear map from $\mathrm{T}_{z} \mathscr{Z}$ to $\mathrm{T}_{f(z)} \mathscr{Z}$. To avoid having to a mention a representative of an equivalence class of curves when we wish to specify a tangent vector, we will often denote points in $\mathrm{T}_{z} \mathscr{Z}$ as ordered pairs $(z, v)$ where the second component represents the equivalence class of curves at the point $z \in \mathscr{Z}$. With this notation, we will also write $\mathrm{T}_{z} f(z, v)=(f(z), \mathrm{D} f(z) v)$.

Let $p: \mathscr{Z} \rightarrow \mathscr{Y}$ and suppose $\mathscr{Z} \subseteq \mathscr{Z}$ is a submanifold such that $p$ restricted to $\mathscr{Z}$ is the identity map, then, for each $z \in \mathscr{Z}$, the linear map $\mathrm{D} p(z)$ is an automorphism of $\mathrm{T}_{z} \mathscr{X}$. The map $\mathscr{D}: \mathrm{T}_{\mathscr{Z}} \mathscr{X} \rightarrow \mathrm{T}_{\mathscr{Z}} \mathscr{X}$, defined 
by $\mathscr{D}(z, v):=(z, \mathrm{D} p(z) v-v)$, is called the infinitesimal displacement of $p$ over $\mathscr{Z}$. We define the kernel of $\mathscr{D}$ by

$$
K \mathscr{D}:=\left\{(z, v) \in \mathrm{T}_{\mathscr{Z}} \mathscr{X}: \mathscr{D}(z, v)=(z, 0)\right\}
$$

and the range of $\mathscr{D}$ by

$$
R \mathscr{D}:=\left\{(z, v) \in \mathrm{T}_{\mathscr{Z}} \mathscr{X}: \mathscr{D}(z, v)=(z, w) \text { for some }(z, w) \in \mathrm{T}_{\mathscr{X}} \mathscr{X}\right\} .
$$

The following proposition is obvious, but fundamental to our approach.

Proposition 2.1. Suppose $\mathscr{Z}$ is an open submanifold of $\mathscr{Y}$. If the map $p$ : $\mathscr{Z} \rightarrow \mathscr{Y}$ is the identity when restricted to the submanifold $\mathscr{Z} \subseteq \mathscr{Z}$ and $\mathscr{D}$ is the infinitesimal displacement of $p$ over $\mathscr{Z}$, then $\mathrm{T} \mathscr{Z} \subseteq K \mathscr{D}$.

Proof. If $(x, v) \in \mathrm{T} \mathscr{Z} \subseteq \mathrm{T}_{\mathscr{Z}} \mathscr{Z}$, then $v$ is represented by a curve $s \mapsto \gamma(s)$ in $\mathscr{Z}$. For this curve $p(\gamma(s))=\gamma(s)$.

Using the notation of this proposition, the submanifold $\mathscr{Z} \subseteq \mathscr{Z}$ is called a normally nondegenerate fixed point manifold for $p$ provided $\mathrm{T} \mathscr{Z}=K \mathscr{D}$. In other words, the submanifold is normally nondegenerate if, at each point of the submanifold, the kernel of the infinitesimal displacement is exactly the tangent space of the submanifold.

Proposition 2.2. If $\mathscr{Z}$ is a normally nondegenerate fixed point manifold for the map $p: \mathscr{Z} \rightarrow \mathscr{Y}$ and $\mathscr{D}$ is the infinitesimal displacement of $p$ over $\mathscr{Z}$, then the range $R \mathscr{D}$ and the kernel $K \mathscr{D}$ are subbundles of $\mathrm{T}_{\mathscr{X}} \mathscr{Z}$. In fact, if $\mathscr{Z}$ has dimension $n$ and $\mathscr{Z}$ has dimension $k$, then $R \mathscr{D}$ has dimension $n$ and fiber dimension $n-k$ while $K \mathscr{D}$ has dimension $2 k$ and fiber dimension $k$. Moreover, for each point $z \in \mathscr{Z}$, there is an open submanifold $Z \subseteq \mathscr{Z}$ containing $z$ and two smooth maps

$$
\mathbf{r}: \mathrm{T}_{Z} \mathscr{X} \rightarrow \mathbb{R}^{n-k} \quad \text { and } \quad \mathrm{s}: \mathrm{T}_{Z} \mathscr{X} \rightarrow \mathbb{R}^{k}
$$

such that the map $\psi: \mathrm{T}_{\mathscr{X}} \mathscr{X} \rightarrow Z \times \mathbb{R}^{n-k} \times \mathbb{R}^{k}$, given by $\psi(\xi, v):=(\xi, \mathbf{r}(\xi, v)$, $\mathbf{s}(\xi, v))$, is an isomorphism. Also, for each $\xi \in \mathscr{Z}$, the restriction $\mathbf{s}: \mathrm{T}_{\xi} \mathscr{Z} \rightarrow \mathbb{R}^{k}$ has kernel $R_{\xi} \mathscr{D}$ and the restriction $\mathbf{r}: R_{\xi} \mathscr{D} \rightarrow \mathbb{R}^{n-k}$ is a linear isomorphism. Proof. Since $\mathscr{Z}$ is normally nondegenerate, the tangent map $\mathrm{T} p: \mathrm{T}_{\mathscr{Z}} \mathscr{X} \rightarrow$ $\mathrm{T}_{\mathscr{X}} \mathscr{X}$ has constant rank $n-k$. The fact that $R \mathscr{D}$ and $K \mathscr{D}$ are subbundles of $\mathrm{T}_{\mathscr{X}} \mathscr{Z}$ with the stated dimensions is a standard result in the theory of vector bundles, for example, see [13, Chapter $3, \S 8]$. The last statement of the theorem follows from the fact that $R \mathscr{D}$ is a subbundle. In fact, there is an open subset $Z \subseteq \mathscr{Z}$ containing the specified point and a local trivialization $\psi: \mathrm{T}_{\mathscr{Z}} \mathscr{Z} \rightarrow$ $Z \times \mathbb{R}^{n-k} \times \mathbb{R}^{k}$ of $T_{\mathscr{X}} \mathscr{Z}$ over $Z$ such that, for each $\xi \in Z$, the restriction $\psi$ : $\mathrm{T}_{\xi} \mathscr{Z} \rightarrow Z \times \mathbb{R}^{n-k} \times \mathbb{R}^{k}$ is a linear isomorphism as is the restriction $\psi: R_{\xi} \mathscr{D} \rightarrow$ $\{\xi\} \times \mathbb{R}^{n-k} \times\{0\}$. The required maps $\mathbf{r}$ and $\mathbf{s}$ are defined, respectively, as the trivialization $\psi$ followed by projection onto the second and third factors of its range.

We say the map $\mathbf{s}$ as in the last proposition complements the range of the infinitesimal displacement of the unperturbed map over $Z \subseteq \mathscr{Z}$.

Suppose $\mathscr{X}, \mathscr{Y}$ and $\mathscr{Z}$ are manifolds, $\mathscr{Y} \subseteq \mathscr{Z}$ is a submanifold and $f: \mathscr{X} \rightarrow \mathscr{Z}$ is a smooth map. Recall that $f$ is called transverse to $\mathscr{Y}$ at a 
point $y \in \mathscr{Y}$ if $y$ is not in the image of $f$ or if $y=f(x)$ and

$$
\mathrm{T}_{x} f\left(\mathrm{~T}_{x} \mathscr{Z}\right)+\mathrm{T}_{f(x)} \mathscr{Y}=\mathrm{T}_{f(x)} \mathscr{Z} .
$$

Let $\mathscr{P}: \mathscr{X} \times \mathbb{R} \rightarrow \mathscr{Y}$ denote a smooth function with associated unperturbed map $p: \mathscr{X} \rightarrow \mathscr{Y}$ and let $\mathscr{Z} \subseteq \mathscr{X}$ denote a normally nondegenerate fixed point manifold for $p$ with associated infinitesimal displacement map $\mathscr{D}$ over $\mathscr{Z}$. For each $z \in \mathscr{Z}$, the curve $\epsilon \mapsto \mathscr{P}(z, \epsilon)$ is in $\mathscr{X}$ with $\mathscr{P}(z, 0)=z$. Thus, this curve defines a tangent vector $P_{\epsilon}(z, 0) \in \mathrm{T}_{z} \mathscr{Z}$. Of course, this vector corresponds to the partial derivative of $\mathscr{P}$ with respect to $\epsilon$.

Define $\mathscr{F}: \mathscr{Z} \rightarrow \mathrm{T}_{\mathscr{Z}} \mathscr{X}$ by

$$
\mathscr{F}(z)=\mathscr{P}_{\epsilon}(z, 0) \text {. }
$$

If $\mathscr{F}(z) \in R \mathscr{D}$ and if $\mathscr{F}$ is transverse to $R \mathscr{D}$ at $\mathscr{F}(z)$, then $z$ is called a transverse unperturbed fixed point of $p$. Also, if $\mathbf{s}: \mathrm{T}_{Z} \mathscr{X} \rightarrow \mathbb{R}^{k}$ is a projection as in Proposition (2.2), we define the associated bifurcation function $\mathscr{B}: Z \rightarrow$ $\mathbb{R}^{k}$ by $\mathscr{B}(z):=\mathbf{s} \mathscr{P}_{\epsilon}(z, 0)$. Finally, $z$ is called a simple zero of a bifurcation function provided $\mathscr{B}(z)=0$ and $\mathrm{T}_{z} \mathscr{B}: \mathrm{T}_{z} Z \rightarrow \mathbb{R}^{k} \times \mathbb{R}^{k}$ is an isomorphism.

Theorem 2.3. Suppose $\mathscr{P}: \mathscr{X} \times \mathbb{R} \rightarrow \mathscr{Y}$ is a smooth function with associated unperturbed map $p: \mathscr{X} \rightarrow \mathscr{Y}$ and $\mathscr{Z} \subseteq \mathscr{X}$ is a normally nondegenerate fuxed point manifold for $p$. If $z \in \mathscr{Z}$ is either a transverse unperturbed fixed point of $p$ or a simple zero of an associated bifurcation function, then $z$ persists.

Proof. If $\mathscr{Z}$ is a $k$ dimensional submanifold of the $n$ dimensional manifold $\mathscr{X}$, then there is an open subset $Z \subseteq \mathscr{Z}$ and a diffeomorphism $\Delta: \mathbb{R}^{k} \times \mathbb{R}^{n-k} \rightarrow$ $Z$, given by $(\theta, \zeta) \mapsto \Delta(\theta, \zeta)$, with $\Delta(0,0)=z$ such that $\theta \mapsto \Delta(\theta, 0)$ is a diffeomorphism with range $Z$. Moreover, there are maps $\mathbf{r}: \mathrm{T}_{\mathscr{Z}} \mathscr{X} \rightarrow \mathbb{R}^{n-k}$ and $\mathbf{s}: \mathrm{T}_{\mathscr{X}} \mathscr{X} \rightarrow \mathbb{R}^{k}$ defined as in Proposition (2.2).

We define the local coordinate representation of $\mathscr{P}$ to be the map $P: \mathbb{R}^{k} \times$ $\mathbb{R}^{n-k} \times \mathbb{R} \rightarrow \mathbb{R}^{k} \times \mathbb{R}^{n-k}$ given by

$$
P(\theta, \zeta, \epsilon)=\Delta^{-1}(\mathscr{P}(\Delta(\theta, \zeta), \epsilon)) .
$$

Note that the displacement $P(\theta, \zeta, \epsilon)-(\theta, \zeta)$ is naturally identified with a tangent vector at the point $(\theta, \zeta) \in \mathbb{R}^{n}$. For a vector such as this in Euclidean space, we follow the usual convention and ignore the base point when we write the vector.

Define $\rho: \mathbb{R}^{k} \times \mathbb{R}^{n-k} \times \mathbb{R} \rightarrow \mathbb{R}^{n-k}$ by

$$
\rho(\theta, \zeta, \epsilon)=\mathbf{r} \mathrm{T} \Delta(P(\theta, \zeta, \epsilon)-(\theta, \zeta)) .
$$

Since, for each $\theta \in \mathbb{R}^{k}$ the point $\Delta(\theta, 0)$ is in the manifold $\mathscr{Z}$ fixed by the unperturbed map, we have $\rho(\theta, 0,0) \equiv 0$. Fix $\theta \in \mathbb{R}^{k}$ and define $w:=$ $\Delta(\theta, 0)$. Also, let $\pi_{2}$ denote the natural projection on the second factor of $\mathbb{R}^{k} \times \mathbb{R}^{n-k}$ and let $I$ denote the identity operator on $\mathrm{T}_{w} \mathscr{Z}$. With this notation, we compute the partial derivative

$$
\begin{aligned}
\rho_{\zeta}(\theta, 0,0) & =\mathbf{r} \mathrm{T} \Delta\left(\left(\mathrm{T}_{w} \Delta\right)^{-1} \mathrm{~T}_{w} p \Delta_{\zeta}(\theta, 0)-\pi_{2}\right) \\
& =\mathbf{r} \mathrm{T}_{w} p \Delta_{\zeta}(\theta, 0)-\mathbf{r} \mathrm{T}_{(\theta, 0)} \Delta \pi_{2} \\
& =\mathbf{r} \mathrm{T}_{w} p \Delta_{\zeta}(\theta, 0)-\mathbf{r} \Delta_{\zeta}(\theta, 0) \\
& =\mathbf{r}\left(\mathrm{T}_{w} p-I\right) \Delta_{\zeta}(\theta, 0) .
\end{aligned}
$$


Since the range of $\Delta_{\zeta}(\theta, 0)$ is complementary to $\mathrm{T}_{w} Z$ in $\mathrm{T}_{w} \mathscr{Z}$ and since $\mathscr{Z}$ is normally nondegenerate

$$
\left(\mathrm{T}_{w} p-I\right) \Delta_{\zeta}(\theta, 0)
$$

is an isomorphism from $\mathbb{R}^{n-k}$ to $R_{w} \mathscr{D}$. Since $\mathbf{r}$ restricted to $R_{w} \mathscr{D}$ is an isomorphism by Proposition (2.2), we see that $\rho_{\zeta}(\theta, 0,0): \mathbb{R}^{n-k} \rightarrow \mathbb{R}^{n-k}$ is an isomorphism. In particular, this partial derivative is an isomorphism at $\theta=0$. Thus, by an application of the Implicit Function Theorem, we conclude there are open subsets $U \subseteq \mathbb{R}^{k}, J \subseteq \mathbb{R}$ and $V \subseteq \mathbb{R}^{n-k}$ with $(0,0) \in U \times J$ and $0 \in V$ together with a smooth map $h: U \times J \rightarrow V$ such that $h(0,0)=0$ and $\rho(\theta, h(\theta, \epsilon), \epsilon) \equiv 0$. Moreover, if $(\theta, \zeta, \epsilon) \in U \times V \times J$ and $\rho(\theta, \zeta, \epsilon)=0$ then $\zeta=h(\theta, \epsilon)$. In particular, since $\rho(\theta, 0,0) \equiv 0$, we have $h(\theta, 0) \equiv 0$.

Define $\tau: U \times J \rightarrow \mathbb{R}^{k}$ by

$$
\tau(\theta, \epsilon)=\mathbf{s} \mathrm{T} \Delta(P(\theta, h(\theta, \epsilon), \epsilon)-(\theta, h(\theta, \epsilon))) .
$$

Since $h(\theta, 0) \equiv 0$, we have $\tau(\theta, 0) \equiv 0$. Thus, there is a smooth function $\mathbf{R}: U \times J \rightarrow \mathbb{R}^{k}$ such that

$$
\tau(\theta, \epsilon)=\epsilon\left(\tau_{\epsilon}(\theta, 0)+\epsilon \mathbf{R}(\theta, \epsilon)\right) .
$$

Of course, here we view the map $\theta \mapsto \tau_{\epsilon}(\theta, 0)$ as a map from $U$ to $\mathbb{R}^{k}$.

If $\tau_{\epsilon}(0,0)=0$ and if $\tau_{\epsilon \theta}(0,0): \mathbb{R}^{k} \rightarrow \mathbb{R}^{k}$ is an isomorphism, then, by an application of the Implicit Function Theorem to the map

$$
(\theta, \epsilon) \mapsto \tau_{\epsilon}(\theta, 0)+\epsilon \mathbf{R}(\theta, \epsilon),
$$

we conclude that there is a curve $\epsilon \mapsto \gamma(\epsilon)$ in $U$ such that $\gamma(0)=0$ and $\tau(\gamma(\epsilon), \epsilon) \equiv 0$. In this case, we have

$$
\begin{aligned}
& \text { s T } \Delta(P(\gamma(\epsilon), h(\gamma(\epsilon), \epsilon), \epsilon)-(\gamma(\epsilon), h(\gamma(\epsilon), \epsilon))) \equiv 0, \\
& \text { rT } \Delta(P(\gamma(\epsilon), h(\gamma(\epsilon), \epsilon), \epsilon)-(\gamma(\epsilon), h(\gamma(\epsilon), \epsilon))) \equiv 0 .
\end{aligned}
$$

In view of Proposition (2.2), $\beta(\epsilon):=\Delta(\gamma(\epsilon), h(\gamma(\epsilon), \epsilon))$ defines a curve in $\mathscr{Z}$ such that $\beta(0)=z$ and $\mathscr{P}(\beta(\epsilon), \epsilon) \equiv \beta(\epsilon)$. In other words, the unperturbed fixed point $z$ persists.

To complete the proof, we will show the transversality of $z$ implies $\tau_{\epsilon}(0,0)$ $=0$ and that $\tau_{\epsilon \theta}(0,0): \mathbb{R}^{k} \rightarrow \mathbb{R}^{k}$ is an isomorphism.

To compute $\tau_{\epsilon}(\theta, 0)$, refer to the definition (3) of $\tau$ and note that $\tau$ is the "product" of two functions. Since the displacement vanishes on $\Delta(\theta, 0)$, the derivative of the product will be $\mathbf{s} R \Delta$ times the partial derivative of the second factor. In view of (2), this derivative is given by

$$
\tau_{\epsilon}(\theta, 0)=\mathbf{s}\left(\mathrm{T}_{w} p-I\right) \Delta_{\zeta}(0,0) h_{\epsilon}(\theta, 0)+\mathrm{s} \mathrm{T} \Delta P_{\epsilon}(\theta, 0) .
$$

Since the range of the infinitesimal displacement is in the kernel of $\mathbf{s}$ by Proposition (2.2), the first summand of (4) vanishes and we find

$$
\begin{aligned}
\tau_{\epsilon}(\theta, 0) & =\mathrm{s} \mathrm{T} \Delta P_{\epsilon}(\theta, 0) \\
& =\mathrm{s} \mathscr{P}_{\epsilon}(\Delta(\theta, 0), 0),
\end{aligned}
$$

that is, $\theta \mapsto \tau_{\epsilon}(\theta, 0)$ is a local representation of an associated bifurcation function. Since $z$ is a transverse unperturbed zero, the point $\left(z, \mathscr{P}_{\epsilon}(z, 0)\right)$ is 
in $R_{z} \mathscr{D}$. Thus, by the choice of $s$, it is clear that $\tau_{\epsilon}(0,0)=0$. If $z$ is a simple zero of this bifurcation function, then $\tau_{\epsilon}(0,0)$ is an isomorphism. This proves the second assertion of the theorem.

By Proposition (2.2) the bundle $\mathrm{T}_{Z} \mathscr{X}$ has the local trivialization $\psi: \mathrm{T}_{\mathscr{Z}} \mathscr{Z}$ $\rightarrow Z \times \mathbb{R}^{n-k} \times \mathbb{R}^{k}$ given by $\psi(\xi, v):=(\xi, \mathbf{r}(\xi, v), \mathbf{s}(\xi, v))$. In these coordinates, $\mathscr{F}$ is given by

$$
(\psi \circ \mathscr{F})(\xi)=\left(\xi, \mathbf{r} \mathscr{P}_{\epsilon}(\xi, 0), \mathbf{s} \mathscr{P}_{\epsilon}(\xi, 0)\right) .
$$

Also, in these coordinates, the tangent space to $R \mathscr{D} \subseteq \mathrm{T}_{Z} \mathscr{X}$ at $\mathscr{F}(z)$ is given by

$$
\mathrm{T}_{z} Z \times\left(\left\{\mathbf{r} \mathscr{P}_{\epsilon}(z, 0)\right\} \times \mathbb{R}^{n-k}\right) \times(\{0\} \times\{0\}) .
$$

Since $\mathscr{F}$ is transverse to $R \mathscr{D}$ at $z$, the image $\mathrm{T} \mathscr{F}(\mathrm{T} \mathscr{Z})$ is represented by

$$
\mathrm{T}_{z} Z \times\left(\left\{\mathbf{r} \mathscr{P}_{\epsilon}(z, 0)\right\} \times\{0\}\right) \times\left(\{0\} \times \mathbb{R}^{k}\right) .
$$

But, this means the derivative of the map $\xi \mapsto(s T \Delta) \mathscr{P}_{\epsilon}(\xi, 0)$ at $\xi=z$ is an isomorphism. In view of equation (5), we conclude that $\tau_{\epsilon}(0,0): \mathbb{R}^{k} \rightarrow \mathbb{R}^{k}$ is an isomorphism.

\section{NORMAL COORDiNATES}

By Theorem (2.3), the persistent unperturbed fixed points on a normally nondegenerate fixed point manifold $\mathscr{Z}$ can be determined as the simple zeros of a bifurcation function. To obtain a suitable bifurcation function, we must first choose a projection $\mathbf{s}$, defined over some submanifold $Z \subseteq \mathscr{Z}$ as in Proposition (2.2), whose kernel is the range of the infinitesimal displacement. For some applications, it is useful to construct this map with respect to tangential and "normal" coordinates over $Z$. We will describe this procedure.

The subbundle $\mathrm{T} \mathscr{Z}$ is complemented in $\mathrm{T}_{\mathscr{Z}} \mathscr{X}$. For example, the subbundle in $\mathrm{T}_{\mathscr{Z}} \mathscr{X}$ given by the orthogonal complement of each fiber of $\mathrm{T} \mathscr{Z}$ with respect to a Riemannian metric on $\mathscr{X}$, is a complementary bundle. However, we want to allow for the nonuniqueness of the complement. Thus, we define $\mathscr{E}^{\text {tan }}:=\mathrm{T} \mathscr{Z}$ and let $\mathscr{E}^{\text {nor }}$ denote an arbitrary complementary subbundle. Then

$$
\mathrm{T}_{\mathscr{X}} \mathscr{X}=\mathscr{E}^{\tan } \oplus \mathscr{E}^{\text {nor }}
$$

and we say $\mathscr{E}^{\tan } \oplus \mathscr{E}^{\text {nor }}$ is a normal splitting of $\mathrm{T} \mathscr{X}$ over $\mathscr{Z}$. For $(z, v) \in$ $\mathrm{T}_{\mathscr{I}} \mathscr{X}$, we will denote the projection to the direct summand $\mathscr{E}^{\tan }$ by $\left(z, v^{\tan }\right)$ and the projection to $\mathscr{E}^{\text {nor }}$ by $\left(z, v^{\text {nor }}\right)$.

Proposition 3.1. If $p: \mathscr{X} \rightarrow \mathscr{Y}$ has a fuxed point manifold $\mathscr{Z} \subseteq \mathscr{X}$ and if $\mathscr{E}^{\tan } \oplus \mathscr{E}^{\text {nor }}$ is a normal splitting of $\mathrm{T} \mathscr{Z}$ over $\mathscr{Z}$, then there are bundle maps $A: \mathscr{E}^{\text {nor }} \rightarrow \mathscr{E}^{\tan }$ and $B: \mathscr{E}^{\text {nor }} \rightarrow \mathscr{E}^{\text {nor }}$ such that the restriction of the derivative of $p$ to $\mathrm{T}_{\mathscr{Z}} \mathscr{X}$, in block matrix form with respect to the normal splitting, is given by $\left(\begin{array}{ll}\begin{array}{l}I \\ 0\end{array} & A \\ 0\end{array}\right)$. Moreover, the infinitesimal displacement $\mathscr{D}$ of $p$ over $\mathscr{Z}$ has block matrix form $\left(\begin{array}{ll}0 & A \\ 0 & B-I\end{array}\right)$ and the range of the infinitesimal displacement is given by

$$
\begin{aligned}
\left\{(z, u, v) \in \mathscr{E}^{\tan } \oplus \mathscr{E}^{\text {nor }}: A(z) w=u \text { and }(B(z)-I) w\right. & =v \\
& \text { for some } \left.(z, w) \in \mathscr{E}^{\text {nor }}\right\} .
\end{aligned}
$$


Proof. The first column of the block matrix representation of $\mathrm{T} p$ has the stated form because $\mathrm{T} p$ is the identity on $\mathrm{T} \mathscr{Z}=\mathscr{E}^{\text {tan }}$.

To compute a bifurcation function, we need a map that complements the range of the infinitesimal displacement. The following theorem gives an explicit formula for such a map in two important special cases.

Theorem 3.2. Suppose $\mathscr{X}$ is an $n$ dimensional manifold, $\mathscr{P}: \mathscr{X} \times \mathbb{R} \rightarrow \mathscr{Y}$, and assume, in addition to the hypotheses and notation of Proposition (3.1), that $\mathscr{Z} \subseteq \mathscr{X}$ is a $k$ dimensional normally nondegenerate fixed point manifold for the associated unperturbed map $p$. Also, suppose there is an open submanifold $Z \subseteq$ $\mathscr{Z}$ and local trivializations $\psi^{\text {tan }}: \mathscr{E}_{Z}^{\tan } \rightarrow Z \times \mathbb{R}^{k}$ and $\psi^{\text {nor }}: \mathscr{E}_{Z}^{\text {nor }} \rightarrow Z \times \mathbb{R}^{n-k}$ given by $(z, u) \mapsto\left(z, \pi^{\tan }(z) u\right)$ and $(z, v) \mapsto\left(z, \pi^{\mathrm{nor}}(z) v\right)$, respectively.

1. If $n=2 k$ and $\mathrm{T} Z$ is the range of the infinitesimal displacement over $X$, equivalently $B-I=0$, then the map given by $(z, v) \mapsto \pi^{\tan }(z) v^{\text {nor }}$ complements the range of $\mathscr{D}$ over $Z$ and the associated bifurcation function for $\mathscr{P}$ over $Z$ is given by

$$
\mathscr{B}(z)=\pi^{\tan }(z) A(z)\left(\mathscr{P}_{\epsilon}(z, 0)\right)^{\text {nor }} .
$$

2. If the restriction of $B-I$ to $\mathscr{E}_{Z}^{\text {nor }}$ is an isomorphism, then the map

$$
(z, v) \mapsto \pi^{\tan }(z)\left(A(z)(B(z)-I)^{-1} v^{\text {nor }}-v^{\tan }\right)
$$

complements the range of $\mathscr{D}$ over $Z$ and the bifurcation function for $\mathscr{P}$ over $Z$ is given by

$$
\mathscr{B}(z)=\pi^{\tan }(z)\left(A(z)(B(z)-I)^{-1}\left(\mathscr{P}_{\epsilon}(z, 0)\right)^{\text {nor }}-\left(\mathscr{P}_{\epsilon}(z, 0)\right)^{\tan }\right) .
$$

Proof. Under the hypotheses of (1), we have $n-k=k$. Also, using Proposition (3.1), we observe that the range of the infinitesimal displacement is given by

$$
\left\{(z, u, 0) \in \mathscr{E}^{\tan } \oplus \mathscr{E}^{\text {nor }}: A(z) w=u \text { for some } \quad(z, w) \in \mathscr{E}^{\text {nor }}\right\} .
$$

Since $\mathscr{Z}$ is normally nondegenerate, the kernel of the infinitesimal displacement is exactly the bundle $\mathscr{E}^{\text {tan }}$ and, as a result, its range is $n=2 k$ dimensional-the fiber dimension is $n-k=k$. It follows that the map $A: \mathscr{E}^{\text {nor }} \rightarrow \mathscr{E}^{\text {tan }}$ is an isomorphism and that the range of the infinitesimal displacement is exactly $\mathscr{E}_{Z}^{\tan }$. Since $\mathscr{E}^{\text {nor }}$ complements $\mathscr{E}^{\tan }$, the map $(z, v) \mapsto \pi^{\tan }(z) A(z) v^{\text {nor }}$ complements the range of $\mathscr{D}$ over $Z$. This proves (1).

Under the hypotheses of (2), the map $B-I$ is an isomorphism. Using Proposition (3.1), it is easy to see that the range of the infinitesimal displacement is given by

$$
\left\{(z, u, v) \in \mathscr{E}^{\tan } \oplus \mathscr{E}^{\text {nor }}: A(z)(B(z)-I)^{-1} v=u\right\} .
$$

In particular, the map

$$
(z, u, v) \mapsto\left(z, A(z)(B(z)-I)^{-1} v-u\right)
$$

has kernel $R \mathscr{D}_{Z}$. Since, by the normal nondegeneracy, this kernel has fiber dimension $n-k$, the induced map on the quotient $\mathscr{E}^{\tan } \oplus \mathscr{E}^{\text {nor }} /(R \mathscr{D})$ is an 
isomorphism. Hence, the map

$$
(z, u, v) \mapsto \pi^{\tan }(z)\left(A(z)(B(z)-I)^{-1} u-v\right)
$$

complements the range of the infinitesimal displacement of $p$ over $Z$.

\section{Persistence of periodic solutions}

In this section we consider a smooth one parameter family of differential equations of the form

$$
\dot{\mathbf{x}}=F(\mathbf{x}, \epsilon), \quad \mathbf{x} \in \mathbb{R}^{n+1}, \quad \epsilon \in \mathbb{R} .
$$

For notational convenience, we define $f: \mathbb{R}^{n+1} \rightarrow \mathbb{R}^{n+1}$ by $f(\mathbf{x}):=F(\mathbf{x}, 0)$. In case the unperturbed system associated to (8), namely $\dot{\mathbf{x}}=f(\mathbf{x})$, has an invariant manifold $\mathscr{A}$ of periodic solutions, we will apply the results of the previous sections to determine which of the periodic solutions on $\mathscr{A}$ persist for $\epsilon \neq 0$.

The link to our general results is provided by the Poincare map. To define this concept, let $t \mapsto \mathbf{U}(t, \nu, \epsilon)$ denote the solution of (8) with the initial value $U(0, \nu, \epsilon)=\nu$. Also, we define $\mathbf{u}(t, \nu):=\mathbf{U}(t, \nu, 0)$ to be the corresponding unperturbed solution. The map $(t, \nu) \mapsto \mathbf{u}(t, \nu)$ is also called the flow of the system $\dot{\mathbf{u}}=f(\mathbf{u})$.

If $\mathscr{S}$ is an $n$ dimensional submanifold of $\mathbb{R}^{n+1}$ transverse to the vector field defined by $f$, then there is an open interval $J \subseteq \mathbb{R}$ containing the origin such that, for each $\epsilon \in J$, the vector field defined by the map $\mathbf{x} \mapsto F(\mathbf{x}, \epsilon)$ is transverse to $\mathscr{S}$. If, in addition, there is a submanifold $\Sigma \subseteq \mathscr{S}$ and a function RT : $\Sigma \times J \rightarrow \mathbb{R}$ such that, for each $(\xi, \epsilon) \in \Sigma \times J$, the point $\mathbf{U}(\operatorname{RT}(\xi, \epsilon), \xi, \epsilon) \in \mathscr{S}$, then $\Sigma$ is called a Poincare section for the parametrized system (8), RT is called the parametrized return time map and $\mathscr{P}: \Sigma \times J \rightarrow \mathscr{S}$, given by

$$
\mathscr{P}(\xi, \epsilon):=\mathbf{U}(\mathbf{R T}(\xi, \epsilon), \xi, \epsilon),
$$

is the parametrized Poincare map. If $\epsilon \in J$, the map $\xi \mapsto \mathscr{P}(\xi, \epsilon)$ is called the Poincare map or the return map for the corresponding differential equation. We define $\mathscr{P}^{1}(\xi, \epsilon):=\mathscr{P}(\xi, \epsilon)$ and, inductively for each integer $N>1$, the map $\left.\mathscr{P}^{N}(\xi, \epsilon):=\mathscr{P}^{\left(\mathscr{P}^{N-1}\right.}(\xi, \epsilon), \epsilon\right)$. If, in addition, we define

$$
\mathbf{R T}^{N}(\xi, \epsilon):=\sum_{i=0}^{N-1} \mathbf{R T}\left(\mathscr{P}^{i}(\xi, \epsilon), \epsilon\right),
$$

then

$$
\mathscr{P}^{N}(\xi, \epsilon)=\mathbf{U}\left(\mathbf{R T}^{N}(\xi, \epsilon), \xi, \epsilon\right) .
$$

It is also convenient to define the (unperturbed) Poincare map $p: \Sigma \rightarrow \mathscr{S}$ by $p(\xi):=\mathscr{P}(\xi, 0)$ and the (unperturbed) return time by $\operatorname{rt}(\xi):=\mathrm{RT}(\xi, 0)$.

By a standard application of the Implicit Function Theorem, it is easy to see that if $\nu \in \mathbb{R}^{n+1}$ lies on a periodic orbit of the unperturbed system, then there is an integer $N \geq 1$ such that $p^{N}(\nu)=\nu$, an open interval $J \subseteq \mathbb{R}$, a section $\mathscr{S}$ and a subsection $\Sigma$ containing $\nu$ such that a parametrized Poincaré map is defined on $\Sigma \times J$. Also, it is clear that, for $\epsilon \in J$, the point $\xi \in \Sigma$ lies on 
a periodic solution of (8) if and only if there is an integer $M \geq 1$ such that $\mathscr{P}^{M}(\xi, \epsilon)=\xi$.

Suppose $\mathscr{A} \subseteq \mathbb{R}^{n+1}$ is a submanifold such that every point of $\mathscr{A}$ lies on a periodic orbit of the unperturbed system. If for every Poincare section $\Sigma$ with $\Sigma \cap \mathscr{A} \neq \varnothing$ and for every point $\xi \in \Sigma \cap \mathscr{A}$ there is a positive integer $N$ such that $p^{N}(\xi)=\xi$, then $\mathscr{A}$ is called a period manifold. If $N$ is the smallest such integer, then $\mathscr{A}$ is called a period manifold of order $N$.

In case $\mathscr{A}$ is a period manifold of order $N$ and $\Sigma$ is a Poincaré section such that $\mathscr{Z}:=\mathscr{A} \cap \Sigma \neq \varnothing$, then $\mathscr{Z}$ is a fixed point manifold for the $N$ th iterate of the unperturbed Poincare map $p$. If, for every Poincaré section that has nonempty intersection with $\mathscr{A}$, the set $\mathscr{Z}$ is a normally nondegenerate fixed point manifold for $p^{N}$, then we say $\mathscr{A}$ is a normally nondegenerate period manifold for $p$ of order $N$. In this case, we can determine the persistent periodic solutions on $\mathscr{A}$ by applying our Lyapunov-Schmidt reduction technique to the map $\mathscr{P}^{N}: \Sigma \rightarrow \mathscr{S}$.

In many applications it is often useful to express the bifurcation function in normal coordinates. For this, recall that the map $\nu \mapsto(\nu, f(\nu))$ defines a vector field on $\mathbb{R}^{n+1}$ that is tangent to $\mathscr{A}$. In particular, this vector field defines a line bundle $\mathscr{E}$ over $\mathscr{A}$. If $\mathscr{E}^{\text {tan }}$ is a bundle complementary to $\mathscr{E}$ in T $\mathscr{A}$ and if $\mathscr{E}^{\text {nor }}$ is a bundle complementary to $\mathscr{E} \oplus \mathscr{E}^{\tan }$ in the tangent bundle of $\mathbb{R}^{n+1}$, then we say $\mathscr{E} \oplus \mathscr{E}^{\tan } \oplus \mathscr{E}^{\text {nor }}$ is a normal splitting over $\mathscr{A}$. Moreover, if $\Sigma$ is a Poincaré section, $\mathscr{Z}:=\mathscr{A} \cap \Sigma \neq \varnothing$, and $\mathrm{T} \mathscr{Z}=\mathscr{E}_{\mathscr{Z}}^{\text {tan }} \oplus \mathscr{E}_{\mathscr{Z}}^{\text {nor }}$, then we say the normal splitting is adapted to $\Sigma$ over $\mathscr{A}$.

In order to use the results of Proposition (3.1) and Theorem (3.2), we must compute the derivative of the unperturbed Poincaré map relative to an adapted normal splitting. This derivative is obtained by solving the first variational equation of the unperturbed system over a solution on the period manifold. More precisely, if $t \mapsto \mathbf{u}(t, \nu)$ is a solution of the unperturbed system, the first variational equation is given by the time dependent linear system

$$
\dot{\mathbf{w}}=\mathrm{D} f(\mathbf{u}(t, \nu)) \mathbf{W} .
$$

Proposition 4.1. Suppose the differential equation $\dot{\mathbf{u}}=f(\mathbf{u}), \mathbf{u} \in \mathbb{R}^{n+1}$ with flow $(t, \nu) \mapsto \mathbf{u}(t, \nu)$ has a period manifold $\mathscr{A}$. If $\mathscr{E} \oplus \mathscr{E}^{\tan } \oplus \mathscr{E}^{\text {nor }}$ is a normal splitting over $\mathscr{A}$, then the principal fundamental matrix solution $\Phi(t, \nu)$ of the first variational equation at $t=0$ defined over the solution $t \mapsto \mathbf{u}(t, \nu)$ is $a$ linear transformation

$$
\Phi(t, \nu):\left(\mathscr{E} \oplus \mathscr{E}^{\tan } \oplus \mathscr{E}^{\text {nor }}\right)_{\nu} \rightarrow\left(\mathscr{E} \oplus \mathscr{E}^{\tan } \oplus \mathscr{E}^{\text {nor }}\right)_{\mathbf{u}(t, \nu)}
$$

with the following block matrix form relative to the normal splitting:

$$
\Phi(t, \nu)=\left(\begin{array}{ccc}
1 & e(t, \nu) & d(t, \nu) \\
0 & c(t, \nu) & a(t, \nu) \\
0 & 0 & b(t, \nu)
\end{array}\right)
$$

The component operators of the block matrix have the following initial values:

$$
e(0, \nu)=0, \quad d(0, \nu)=0, \quad c(0, \nu)=I, \quad a(0, \nu)=0, \quad b(0, \nu)=I .
$$


In addition, the inverse fundamental matrix, with the argument $(t, \nu)$ of its component operators suppressed, is given by

$$
\Phi^{-1}(t, \nu)=\left(\begin{array}{ccc}
1 & -e c^{-1} & -d b^{-1}+e c^{-1} a b^{-1} \\
0 & c^{-1} & -c^{-1} a b^{-1} \\
0 & 0 & b^{-1}
\end{array}\right) .
$$

Proof. The structure of the first column of $\Phi(t, \nu)$ is a direct consequence of the fact that $w(t):=f(\mathbf{u}(t, \nu))$ is a solution of the first variational equation with initial value $w(0)=f(\nu)$. In effect, since the solutions of this differential equation are unique, we have $\Phi(t, \nu) f(\nu)=f(\mathbf{u}(t, \nu))$. The structure of the first column now follows because the vector field defined by $f$ generates the line bundle $\mathscr{E}$.

The structure of the second column is a direct consequence of the fact that the bundle $\mathscr{E} \oplus \mathscr{E}$ tan is invariant under the tangent of the flow $\mathbf{u}$. To see this, note that for $\nu \in \mathscr{A}$, the tangent space $\mathrm{T}_{\nu} \mathscr{A}$ coincides with $(\mathscr{E} \oplus \mathscr{E} \text { tan })_{\nu}$. In particular, if $(\nu, v) \in \mathrm{T}_{\nu} \mathscr{A}$, then there is a curve $s \mapsto \gamma(s)$ in $\mathscr{A}$ such that $\gamma(0)=\nu$ and $\dot{\gamma}(0)=v$. Also, it is easy to see that

$$
\Phi(t, \nu) v=\left.\frac{d}{d s} \mathbf{u}(t, \gamma(s))\right|_{s=0} .
$$

In fact, the function defined by $w(t):=\left.\frac{d}{d s} \mathbf{u}(t, \gamma(s))\right|_{s=0}$ is such that $w(0)=v$ and

$$
\begin{aligned}
\dot{w}(t) & =\left.\frac{d}{d s} \dot{\mathbf{u}}(t, \gamma(s))\right|_{s=0} \\
& =\left.\frac{d}{d s} f(\mathbf{u}(t, \gamma(s)))\right|_{s=0} \\
& =\left.\mathrm{D} f(\mathbf{u}(t, \gamma(s))) \frac{d}{d s} \mathbf{u}(t, \gamma(s))\right|_{s=0} \\
& =\mathrm{D} f(\mathbf{u}(t, \gamma(s))) w(t) .
\end{aligned}
$$

Again, by the uniqueness of the solutions of the first variational equation, we see that $w(t)=\Phi(t, \nu) v$, as required. Since $\gamma$ defines a curve in $\mathscr{A}$, we have, for each $t \in \mathbb{R}$, that the curve $s \mapsto \mathbf{u}(t, \gamma(s))$ is also a curve in $\mathscr{A}$. Thus, by equation (12), it follows that $\Phi(t, \nu) v$ is tangent to $\mathscr{A}$.

The fact that $\Phi(t, \nu)$ is invertible is a standard result in linear systems theory. The block form of the inverse given in the statement of the proposition can be verified by matrix multiplication.

Proposition 4.2. Suppose, in addition to the hypotheses of Proposition (4.1), that the system $\dot{\mathbf{u}}=f(\mathbf{u})$ has a period manifold $\mathscr{A}$ of order $N$ and $\mathscr{E} \oplus \mathscr{E}^{\text {tan }} \oplus \mathscr{E}^{\text {nor }}$ is a normal splitting over $\mathscr{A}$ adapted to a Poincaré section $\Sigma$ with Poincaré map $p$ and return time $\mathrm{rt}$. If $\mathscr{Z}:=\mathscr{A} \cap \Sigma$, then for each $z \in \mathscr{Z}$, the derivative $\mathrm{T}_{z} p^{N}$, in block matrix form relative to $\left(\mathscr{E}^{\mathrm{tan}} \oplus \mathscr{E}^{\mathrm{nor}}\right)_{z}$, is given by

$$
\mathrm{T}_{z} p^{N}=\left(\begin{array}{ll}
I & a\left(\mathrm{rt}^{N}(z), z\right) \\
0 & b\left(\mathrm{rt}^{N}(z), z\right)
\end{array}\right) .
$$


In particular, the bundle maps $A: \mathscr{E}^{\text {nor }} \rightarrow \mathscr{E}^{\text {tan }}$ and $B: \mathscr{E}^{\text {nor }} \rightarrow \mathscr{E}^{\text {nor }}$ in Proposition (4.1) are given by $A(z, v):=\left(z, a\left(\mathrm{rt}^{N}(z), z\right) v\right)$ and $B(z, v):=$ $\left(z, b\left(\mathrm{rt}^{N}(z), z\right) v\right)$.

Proof. Suppose $(z, w)$ is a vector in $\mathrm{T}_{z} \Sigma$ and let $s \mapsto \gamma(s)$ denote a curve in $\Sigma$ tangent to this vector. Using (12) and the fact that $u\left(\mathrm{rt}^{N}(z), z\right)=z$, we compute

$$
\begin{aligned}
\mathrm{D} p^{N}(z) w & =\left.\frac{d}{d s} \mathbf{u}\left(\mathrm{rt}^{N}(\gamma(s)), \gamma(s)\right)\right|_{s=0} \\
& =\dot{\mathbf{u}}\left(\mathrm{rt}^{N}(z), z\right) \mathrm{Drt}^{N}(z) w+\Phi\left(\mathrm{rt}^{N}(z), z\right) w \\
& =f(z) \mathrm{Drt}^{N}(z) w+\Phi\left(\mathrm{rt}^{N}(z), z\right) w .
\end{aligned}
$$

Since $\mathrm{rt}^{N}: \Sigma \rightarrow \mathbb{R}$, the image of the linear map $\mathrm{Drt}^{N}(z)$ is in $\mathbb{R}$. Thus, the first summand of (14) may be viewed as a scalar multiple of $f(z)$. In particular, $\left(z, f(z) \mathrm{Drt}^{N}(z) w\right) \in \mathscr{E}_{z}$. Since $p^{N}: \Sigma \rightarrow \mathscr{S}$ and $p^{N}(z)=z$, the derivative $\mathrm{T}_{z} p^{N}$ is a linear automorphism of $\mathrm{T}_{z} \Sigma=\left(\mathscr{E}^{\tan } \oplus \mathscr{E}^{\mathrm{nor}}\right)_{z}$. Thus, the first component of the second summand of (14) must be $\left(z,-f(z) \mathrm{Drt}^{N}(z) w\right) \epsilon$ $\mathscr{E}_{z}$. In view of Proposition (3.1), these two facts imply the block matrix form of $\mathrm{D} p^{N}(z)$ relative to $\left(\mathscr{E}^{\tan } \oplus \mathscr{E}^{\text {nor }}\right)_{z}$ is given by

$$
\left(\begin{array}{cc}
c\left(\mathrm{rt}^{N}(z), z\right) & a\left(\mathrm{rt}^{N}(z), z\right) \\
0 & b\left(\mathrm{rt}^{N}(z), z\right)
\end{array}\right) .
$$

Finally, since $p^{N}$ is the identity map on $\mathscr{Z}$, the restriction of its derivative to $\mathrm{T} \mathscr{Z}=\mathscr{E}^{\tan }$ is the identity linear transformation, that is $c\left(\mathrm{rt}^{N}(z), z\right)=I$.

Theorem 4.3. Suppose, in addition to the hypotheses of Proposition (4.2), that $\mathscr{A}$ is a $k+1$ dimensional normally nondegenerate period manifold of order $N$ for the unperturbed system $\dot{\mathbf{u}}=f(\mathbf{u}), \mathbf{u} \in \mathbb{R}^{n+1}$ associated with (8). If $Z \subseteq \mathscr{Z}=$ $\mathscr{A} \cap \Sigma$ is an open submanifold, the map $\mathbf{s}: \mathrm{T}_{Z} \Sigma \rightarrow \mathbb{R}^{k}$ complements the range of the unperturbed Poincare map $p$ over $Z$ and if the function $\mathbf{F}: Z \rightarrow \mathscr{E}^{\tan } \oplus \mathscr{E}^{\text {nor }}$ is defined by $\mathbf{F}(z)=(z, \mathscr{N}(z), \mathscr{M}(z))$ where

$$
\begin{aligned}
\mathscr{M}(z):= & \int_{0}^{\mathrm{r}^{N}(z)} b^{-1}(t, z) F_{\epsilon}^{\mathrm{nor}}(\mathbf{u}(t, z), 0) d t \\
\mathscr{N}(z):= & \int_{0}^{\mathrm{r}^{N}(z)} c^{-1}(t, z) F_{\epsilon}^{\mathrm{tan}}(\mathbf{u}(t, z), 0) \\
& -c^{-1}(t, z) a(t, z) b^{-1}(t, z) F_{\epsilon}^{\mathrm{nor}}(\mathbf{u}(t, z), 0) d t
\end{aligned}
$$

then the associated bifurcation function is given by $z \mapsto \mathbf{s F}(z)$ and

$$
\left(\mathscr{P}_{\epsilon}(z, 0)\right)^{\tan }=\mathscr{N}(z)+A(z) \mathscr{M}(z), \quad\left(\mathscr{P}_{\epsilon}(z, 0)\right)^{\text {nor }}=B(z) \mathscr{M}(z) .
$$

Moreover, if the dimension of $\Sigma$ is $n=2 k$ and if $B$, given in Proposition (4.2), is the identity, then the bifurcation function is given by $z \mapsto \pi^{\tan } A(z) \mathscr{M}(z)$. If the restriction of $B-I$ to $\mathscr{E}_{Z}^{\text {nor }}$ is an isomorphism, then the bifurcation function is given by

$$
z \mapsto \pi^{\tan }\left(A(z)(B(z)-I)^{-1} \mathscr{M}(z)-\mathscr{N}(z)\right)
$$


Proof. By Theorem (2.3), it suffices to show $z \mapsto \mathbf{s F}(z)$ is a bifurcation function. To this end, as in (13), we compute

$$
\mathscr{P}_{\epsilon}^{N}(z, 0)=F(z, 0) \mathrm{RT}_{\epsilon}^{N}(z, 0)+\mathrm{U}_{\epsilon}\left(\mathrm{rt}^{N}(z), z, 0\right) .
$$

Since $t \mapsto \mathbf{U}(t, z, \epsilon)$ is the solution of the differential equation (8) and since $\mathrm{U}(0, z, \epsilon)=z$, the partial derivative $t \mapsto \mathbf{U}_{\epsilon}(t, z, 0)$ is the solution of the following initial value problem for the second variational equation:

$$
\dot{\mathbf{V}}=\mathrm{D} f(\mathbf{u}(t, z)) \mathbf{V}+F_{\epsilon}(\mathbf{u}(t, z), 0), \quad \mathbf{V}(0)=0 \text {. }
$$

The solution of this initial value problem is obtained by variation of parameters with respect to the principal fundamental matrix solution $\Phi(t, z)$ of the first variational equation. In fact, we compute

$$
\mathrm{U}_{\epsilon}\left(\mathrm{rt}^{N}(z), z, 0\right)=\Phi\left(\mathrm{rt}^{N}(z), z\right) \int_{0}^{\mathrm{rt}^{N}(z)} \Phi^{-1}(t, z) F_{\epsilon}(\mathbf{u}(t, z), 0) d s .
$$

From (15), as in the proof of Proposition (4.2), $\left(z, F(z, 0) \mathrm{RT}_{\epsilon}^{N}(z, 0)\right) \in \mathscr{E}_{z}$ and $\mathscr{P}_{\epsilon}^{N}(z, 0) \in\left(\mathscr{E}^{\tan } \oplus \mathscr{E}^{\text {nor }}\right)_{z}$. Thus, the first component, relative to the normal splitting, of the second summand of $(15)$ is $\left(z,-F(z, 0) \mathrm{RT}_{\epsilon}^{N}(z, 0)\right) \epsilon$ $\mathscr{E}_{z}$. Using these facts, Proposition (4.2), and the block matrix representations of $\Phi(t, z)$ and its inverse given in Proposition (4.1), a simple computation shows the block matrix representation of $\mathscr{P}_{\epsilon}^{N}(z, 0)$ is

$$
\mathscr{P}_{\epsilon}^{N}(z, 0)=\left(\begin{array}{ll}
I & A(z) \\
0 & B(z)
\end{array}\right)\left(\begin{array}{l}
\mathscr{N}(z) \\
\mathscr{M}(z)
\end{array}\right)
$$

for $\mathscr{M}$ and $\mathscr{M}$ as in the statement of the proposition.

Observe that

$$
\mathscr{P}_{\epsilon}^{N}(z, 0)=\left(\begin{array}{c}
\mathscr{N}(z) \\
\mathscr{M}(z)
\end{array}\right)+\left(\begin{array}{c}
A(z) \mathscr{M}(z) \\
(B(z)-I) \mathscr{M}(z)
\end{array}\right)
$$

By Proposition (3.1) the second summand of the right hand side of (16) is in the range of the infinitesimal displacement of the unperturbed Poincare map over $Z$. Thus, the bifurcation function, $z \mapsto \operatorname{s}_{\epsilon}^{N}(z, 0)$ reduces to $z \mapsto \mathbf{s F}(z)$ as in the statement of the proposition.

The last two statements of the proposition follow from Theorem (3.2). We have just proved $\left(\mathscr{P}_{\epsilon}^{N}\right)^{\tan }=\mathscr{N}+A \mathscr{M}$ and $\left(\mathscr{P}_{\epsilon}^{N}\right)^{\text {nor }}=B \mathscr{M}$. In the first case, that is in case $B=I$, the result is immediate from statement (1) of Theorem (3.2). For the second case, we note that $(B-I)^{-1} B-I=(B-I)^{-1}$ and, using this identity, we find

$$
A(B-I)^{-1} B \mathscr{M}-(\mathscr{N}+A \mathscr{K})=A(B-I)^{-1} \mathscr{M}-\mathscr{N} .
$$

The desired result is then an immediate consequence of statement (2) of Theorem (3.2).

The function $\mathscr{M}$ is a generalization of the "Melnikov integral". The reader unfamiliar with this notion is referred to the original paper of Melnikov [14] and the books [12], [15]. Also, for specializations of the theory presented in this paper to periodically forced nonlinear oscillators see [5] and for specializations to coupled systems of nonlinear oscillators see [6], [7]. 


\section{Periodic solutions of ABC systems}

The equations of motion of an ideal fluid in a three dimensional bounded region are given by Bernoulli's form of Euler's equations as

$$
\frac{\partial V}{\partial t}=V \wedge \operatorname{curl} V+\operatorname{grad} \alpha, \quad \operatorname{div} V=0,
$$

where $V$ denotes the velocity of the fluid and $\alpha$ is a function determined by the fact that $\operatorname{div} V=0$ and the additional condition that $V$ is tangent to the boundary of the region. For the idealized case of steady flow on the three torus $\mathbb{T}^{3}$, the boundary in empty. Thus, we can take $\alpha=0$ and seek "curl parallel" [4] steady state solutions on $\mathbb{T}^{3}$, that is, solutions of $V \wedge \operatorname{curl} V=0$. The vector field on $\mathbb{T}^{3}$ associated with the $A B C$ system,

$$
\begin{aligned}
& \dot{x}=A \sin z+C \cos y, \\
& \dot{y}=B \sin x+A \cos z, \\
& \dot{z}=C \sin y+B \cos x,
\end{aligned}
$$

where the real coordinates $x, y$ and $z$ are viewed modulo $2 \pi$, is curl parallel.

This system was introduced by V. I. Arnold [1] and studied recently in [9] with regard to the hydrodynamic instability criterion of S. Friedlander and M. M. Vishik [10], [11]. A special case of their theory shows that the existence of a hyperbolic periodic solution of an $\mathrm{ABC}$ system implies the hydrodynamic instability of the associated steady state solution of Euler's equation. The main result of [9] is the following perturbation theorem:

Theorem 5.1. Consider (17) with $A=1$ and $\epsilon:=B=C$. If $\epsilon>0$ is sufficiently small, then (17) has a hyperbolic periodic solution.

In the first subsection we will apply our general results to give a new proof of Theorem (5.1). In the second subsection we will study the more difficult case where there is only one small parameter. We will determine the number and position of the persistent unperturbed periodic solutions. However, one of the main results can be easily formulated as the following theorem:

Theorem 5.2. Consider (17) with $A=1,0<B \leq 1$ and $\epsilon:=C$. If $N$ is $a$ positive integer, then there is some $\epsilon>0$ such that (17) has at least $N$ hyperbolic periodic solutions.

5.1. ABC systems with two small coefficients. By rescaling time and by the symmetries of the $\mathrm{ABC}$ system, there is no loss of generality if we assume $A=1$. We will show the system

$$
\begin{aligned}
& \dot{x}=\sin z+\epsilon \gamma \cos y, \\
& \dot{y}=\cos z+\epsilon \beta \sin x, \\
& \dot{z}=\quad \epsilon(\gamma \sin y+\beta \cos x)
\end{aligned}
$$

with $\beta \gamma \neq 0$, has periodic solutions for $\epsilon \neq 0$ and sufficiently small. We will not take the most efficient route to determine the persistent periodic solutions. Rather, we will use this problem to illustrate the general theory developed above. 
The flow of the unperturbed system is given by

$$
\mathbf{u}(t, x, y, z):=(x+t \sin z, y+t \cos z, z)
$$

and

$$
\mathscr{S}:=\left\{(x, y, z) \in \mathbb{T}^{3}: y=0 \text { and } z \neq \pi / 2\right\}
$$

is a Poincaré section for this flow. In fact, the return time is given by $\operatorname{rt}(x, z)=$ $2 \pi / \cos z$ and the unperturbed Poincaré map $p^{N}: \mathscr{S} \rightarrow \mathscr{S}$ is given by

$$
p(x, z)=(x+2 \pi N \tan z, z) \text {. }
$$

If there are integers $M$ and $N>0$ such that $\tan z=M / N$, then

$$
\mathscr{A}:=\{(x, z) \in \mathscr{S}: \tan z=M / N\}
$$

is a period manifold (in this case a two dimensional torus) of order $N$. In this context, $\mathscr{A}$ is also called a resonant torus.

To check the normal nondegeneracy of $\mathscr{A}$, we note that the usual coordinates of $\mathbb{R}^{2}$ provide a normal splitting over each resonant torus and that the derivative of the unperturbed Poincare map is represented by its Jacobian matrix as follows:

$$
\mathrm{D} p^{N}(x, z)=\left(\begin{array}{cc}
1 & 2 \pi N \sec ^{2} z \\
0 & 1
\end{array}\right)
$$

Also, if $(x, z) \in \mathscr{S}$, then $\cos z \neq 0$. Thus, the principal part of the infinitesimal displacement $\mathscr{D}$, namely

$$
\mathrm{D} p^{N}(x, z)-I=\left(\begin{array}{cc}
0 & 2 \pi N \sec ^{2} z \\
0 & 0
\end{array}\right),
$$

has kernel and range given by

$$
K=R=\left[\begin{array}{l}
1 \\
0
\end{array}\right]
$$

where here and hereafter we let square brackets denote the span of the enclosed vector. The bundle $K \mathscr{D}=R \mathscr{D}$ is, of course, the trivial bundle $\mathscr{Z} \times K$. Since $\mathscr{Z}=\mathscr{A} \cap \mathscr{S}$ is one dimensional, it follows that each resonant torus is normally nondegenerate.

Here, the complement of the range $R$ is

$$
C R=\left[\begin{array}{l}
0 \\
1
\end{array}\right] \text {. }
$$

Thus, a bundle map $s$ that complements the range of the infinitesimal displacement is given by projection to $C R$ in the usual coordinates of $\mathbb{R}^{2}$.

If we define $t \mapsto \mathrm{U}(t, x, y, z, \epsilon)$ to be the solution of (18) with initial condition $\mathrm{U}(0, x, y, z, \epsilon)=(x, y, z)$, then there is a parametrized return time map $R T: \Sigma \times \mathbb{R} \rightarrow \mathbb{R}$ and the parametrized Poincare map for the system (18) is defined on some open subset $\Sigma \subseteq \mathscr{S}$ by

$$
\mathscr{P}(x, z, \epsilon)=\mathbf{U}(\mathbf{R T}(x, z, \epsilon), x, 0, z, \epsilon)) .
$$

Recall that the bifurcation function is $(x, z) \rightarrow s \mathscr{P}_{\epsilon}(x, z, 0)$ Thus, we must compute $\mathscr{P}_{\epsilon}(x, z, 0)$. 
The partial derivative $t \mapsto \mathbf{U}_{\epsilon}(t, x, y, z, 0)$ is the solution of the second variational initial value problem

$$
\dot{\mathbf{W}}=\left(\begin{array}{ccc}
0 & 0 & \cos z \\
0 & 0 & -\sin z \\
0 & 0 & 0
\end{array}\right) \mathbf{W}+\left(\begin{array}{l}
\gamma \cos (t \cos z) \\
\beta \sin (x+t \sin z) \\
\gamma \sin (t \cos z)+\beta \cos (x+t \sin z)
\end{array}\right), \quad \mathbf{W}(0)=0 .
$$

We can, of course, solve this initial value problem directly. For this, note that the principal fundamental matrix at $t=0$ is given by

$$
\Phi(t, x, z)=\left(\begin{array}{ccc}
1 & 0 & t \cos z \\
0 & 1 & -t \sin z \\
0 & 0 & 1
\end{array}\right)
$$

and use variation of parameters to obtain

$$
\begin{gathered}
\mathbf{W}(2 \pi N / \cos z)=\left(\begin{array}{llc}
1 & 0 & 2 \pi N \\
0 & 1 & -2 \pi N \tan z \\
0 & 0 & 1
\end{array}\right) \\
\times\left(\begin{array}{c}
\int_{0}^{2 \pi N} \gamma \cos (s)-s(\gamma \sin (s)+\beta \cos (x+M s / N)) d s \\
\int_{0}^{2 \pi N} \beta \sin (x+M s / N)-s(\gamma \sin (s)+\beta \cos (x+M s / N)) \sec z d s \\
\int_{0}^{2 \pi N} \gamma \sin (s)+\beta \cos (x+M s / N) \sec z d s
\end{array}\right) .
\end{gathered}
$$

If $M \neq 0$, then

$$
\begin{aligned}
\mathbf{W}(2 \pi N / \cos z) & =\left(\begin{array}{ccc}
1 & 0 & 2 \pi N \\
0 & 1 & -2 \pi N \tan z \\
0 & 0 & 1
\end{array}\right)\left(\begin{array}{c}
2 \pi N \gamma-(2 \pi N \beta \sin x) N / M \\
2 \pi N \beta \sin x-2 \pi M \gamma \\
0
\end{array}\right) \\
& =\left(\begin{array}{c}
2 \pi N \gamma-(2 \pi N \beta \sin x) N / M \\
2 \pi N \beta \sin x-2 \pi M \gamma \\
0
\end{array}\right) .
\end{aligned}
$$

If $M=0$, then

$$
\begin{aligned}
\mathbf{W}(2 \pi N \sec z) & =\left(\begin{array}{ccc}
1 & 0 & 2 \pi N \\
0 & 1 & -2 \pi N \tan z \\
0 & 0 & 1
\end{array}\right)\left(\begin{array}{c}
2 \pi N \gamma-2 \pi^{2} N^{2} \beta \cos x \\
2 \pi N \beta \sin x \sec z \\
2 \pi N \beta \cos x \sec z
\end{array}\right) \\
& =\left(\begin{array}{c}
2 \pi N \gamma-2 \pi^{2} N^{2} \beta \cos x(2 \sec z-1) \\
2 \pi N \beta \sec z(\sin x-2 \pi N \cos x \tan z) \\
2 \pi N \beta \cos x \sec z
\end{array}\right) .
\end{aligned}
$$

However, to illustrate our theory, we will find a bifurcation function relative to a normal splitting using the formulas developed previously. For this, we express the principal fundamental matrix $\Phi(t, x, z)$ as a block matrix relative to the adapted normal splitting over a fixed resonant torus $\mathscr{A}$ (fixed $z$ ) given by

$$
\mathscr{E}:=\mathscr{A} \times\left[\begin{array}{c}
\sin z \\
\cos z \\
0
\end{array}\right], \quad \mathscr{E}^{\text {tan }}:=\mathscr{A} \times\left[\begin{array}{l}
1 \\
0 \\
0
\end{array}\right], \quad \mathscr{E}^{\text {nor }}:=\mathscr{A} \times\left[\begin{array}{l}
0 \\
0 \\
1
\end{array}\right] .
$$


Note that $x$ gives the local coordinate on $\mathscr{A}$ and compute

$$
\Phi(t, x)=\left(\begin{array}{llc}
1 & 0 & -t \tan z \\
0 & 1 & t(\cos z+\tan z \sin z) \\
0 & 0 & 1
\end{array}\right)
$$
by

From (19) we find $B=I$ while $A$ is the operator of scalar multiplication

$$
(2 \pi N / \cos z)(\cos z+\tan z \sin z)=2 \pi N \sec ^{2} z .
$$

More precisely, the principal part of the operator $A$ is given by

$$
\zeta\left(\begin{array}{l}
0 \\
0 \\
1
\end{array}\right) \mapsto 2 \pi N \zeta \sec ^{2} z\left(\begin{array}{l}
1 \\
0 \\
0
\end{array}\right) \text {. }
$$

The $\mathscr{E}^{\text {nor }}$ component of the perturbation term of the system (18) along the unperturbed solution is given by

$$
(c \sin (t \cos z)+\beta \cos (x+t \sin z))\left(\begin{array}{l}
0 \\
0 \\
1
\end{array}\right) .
$$

By Theorem (4.3) we have

$$
\mathscr{M}(x)=\int_{0}^{2 \pi N / \cos z} \gamma \sin (t \cos z)+\beta \cos (x+t \sin z) d t\left(\begin{array}{l}
0 \\
0 \\
1
\end{array}\right)
$$

and the associated bifurcation function $x \mapsto \pi^{\tan } A(z) \mathscr{M}(z)$ is given by

$$
x \mapsto \begin{cases}(2 \pi N)^{2} \beta \sec ^{2}(z) \cos x / \cos z, & \text { if } M=0, \\ 0, & \text { if } M \neq 0 .\end{cases}
$$

In case $M=0$, recall (18) and note that we must have $z=0$ or $z=\pi$. Thus, the bifurcation function is given by $x \mapsto \pm(2 \pi N)^{2} \beta \cos x$. In particular, the bifurcation function has simple zeros at $x=\pi / 2$ and $x=3 \pi / 2$ that correspond to persistent unperturbed periodic solutions. The same persistent periodic solutions are obtained in [9]. In case $M \neq 0$, the bifurcation function vanishes identically and our first order theory does not determine the persistent periodic solutions.

The stability of the perturbed periodic solutions is determined by computing the spectrum of the derivative of the Poincaré map $(x, z) \mapsto \mathscr{P}^{N}(x, z, \epsilon)$ at the intersection of the perturbed periodic solution with the Poincare section. Of course, since we do not know this point exactly, it is natural to compute the derivative at the persistent unperturbed solution using a regular perturbation series. Here, for arbitrary $(x, z) \in \Sigma$, we have

$$
\mathrm{D} \mathscr{P}^{N}(x, z, \epsilon)=\mathrm{D} p^{N}(x, z)+\epsilon \mathrm{D} \mathscr{P}_{\epsilon}^{N}(x, z, 0)+O\left(\epsilon^{2}\right) .
$$

We must exercise caution [15, pp. 140-143] when approximating the eigenvalues of the linearized Poincaré map from this regular perturbation series. However, in the present case, we can obtain the stability results by using the fact that we know $\mathrm{D} p^{N}(x, z)$ explicitly. 
In normal coordinates,

$$
\mathrm{D} p^{N}(x, z)=\left(\begin{array}{cc}
1 & 2 \pi N \sec ^{2} z \\
0 & 1
\end{array}\right)
$$

Also, if the functions $p_{i j}$ are defined by the equation

$$
\mathrm{D} \mathscr{P}_{\epsilon}^{N}(x, z, 0)=\left(\begin{array}{ll}
p_{11}(x, z) & p_{12}(x, z) \\
p_{21}(x, z) & p_{22}(x, z)
\end{array}\right),
$$

then we have

$$
\begin{aligned}
\operatorname{trace} \mathrm{D} \mathscr{P}^{N}(x, z, \epsilon)= & 2+\epsilon\left(p_{11}(x, z)+p_{22}(x, z)\right)+O\left(\epsilon^{2}\right), \\
\operatorname{det} \mathrm{D} \mathscr{P}^{N}(x, z, \epsilon)= & 1+\epsilon\left(p_{11}(x, z)+p_{22}(x, z)\right) \\
& -\epsilon 2 \pi N p_{21}(x, z) \sec ^{2} z+O\left(\epsilon^{2}\right) .
\end{aligned}
$$

If the persistent periodic solution corresponds to $\left(x_{0}, z_{0}\right) \in \Sigma$, then the perturbed solution corresponds to $\left(x_{0}, z_{0}\right)+O(\epsilon)$. When this formula is substituted into the expressions for the trace and the determinant, and the resulting functions are expanded in powers of $\epsilon$, we see that the Taylor series for the trace and the determinant at the perturbed point are given by

$$
\begin{aligned}
\operatorname{trace} \mathscr{D}^{N}(x, z, \epsilon)= & 2+\epsilon\left(p_{11}\left(x_{0}, z_{0}\right)+p_{22}\left(x_{0}, z_{0}\right)\right)+O\left(\epsilon^{2}\right), \\
\operatorname{det} \mathscr{P}^{N}(x, z, \epsilon)= & 1+\epsilon\left(p_{11}\left(x_{0}, z_{0}\right)+p_{22}\left(x_{0}, z_{0}\right)\right) \\
& -\epsilon 2 \pi N p_{21}\left(x_{0}, z_{0}\right) \sec ^{2} z+O\left(\epsilon^{2}\right) .
\end{aligned}
$$

In other words, it suffices to compute $p_{i j}$ at the unperturbed persistent periodic solution.

Using the quadratic formula, it is easy to see that the eigenvalues of the derivative of the Poincare map at the perturbed solution are given by

$$
1 \pm \sqrt{\epsilon}\left(2 \pi N p_{21}\left(x_{0}, z_{0}\right) \sec ^{2} z\right)^{1 / 2}+\epsilon\left(p_{11}\left(x_{0}, z_{0}\right)+p_{22}\left(x_{0}, z_{0}\right)\right) / 2+O\left(\epsilon^{3 / 2}\right) \text {. }
$$

The stability can always be determined from this expression if both the $O(\sqrt{\epsilon})$ and the $O(\epsilon)$ terms are nonzero. If $p_{21}\left(x_{0}, z_{0}\right)>0$, then the stability, for $\epsilon>0$, is determined by the $O(\sqrt{\epsilon})$ term. In fact, in this case, for sufficiently small $\epsilon>0$, the perturbed periodic point is a hyperbolic saddle.

From (14) and the fact that $\mathscr{M}\left(x_{0}\right)=0$ we compute

$$
p_{21}\left(x_{0}, z_{0}\right)=\mathscr{P}_{\epsilon}^{\text {nor }}\left(x_{0}, z_{0}\right)=B\left(x_{0}\right) \mathscr{M}_{x}\left(x_{0}\right) .
$$

In the case at hand, $B=I, M=0$ and, from (20),

$$
p_{21}\left(x_{0}, z_{0}\right)=-2 \pi N \beta \sin x_{0} .
$$

Thus, the points $(x, z)=(3 \pi / 2,0)$ and $(x, z)=(\pi / 2, \pi)$ are hyperbolic saddle points. This agrees with the computations in [9].

5.2. ABC systems with one small coefficient. In this section we will consider the $\mathrm{ABC}$ system with one small parameter. As in the previous section, we will assume $A=1$ and that $C=\epsilon$, where $\epsilon$ is a small parameter. But, in this section, we will assume the coefficient $\beta:=B$ is a fixed number in the range $0<\beta \leq 1$. Also, in anticipation of some of the computations to follow and 


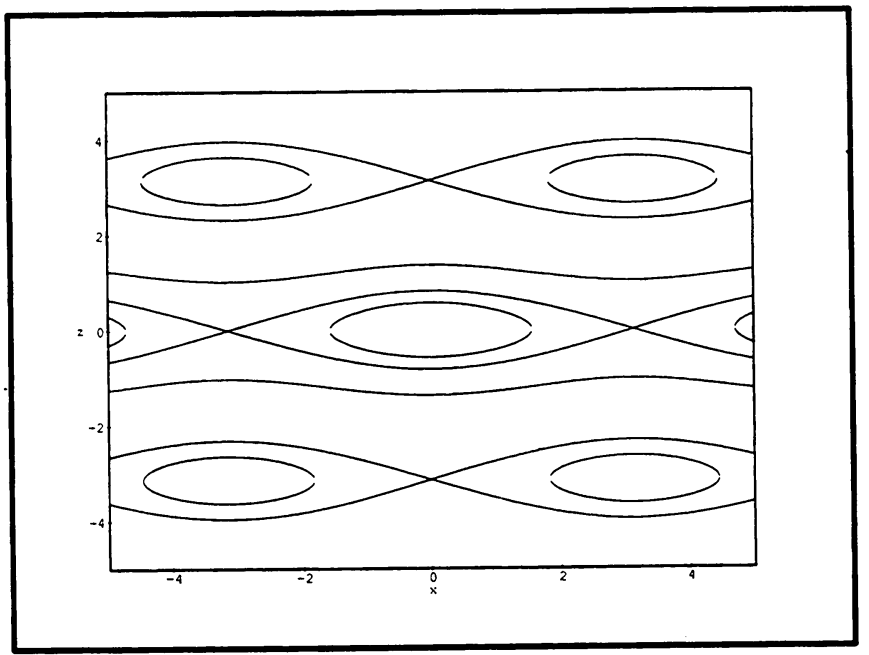

FIGURE 1. Computer generated phase portrait for the Hamiltonian system $\dot{x}=\sin z, \dot{z}=-\beta \sin x$ with $\beta=0.16$.

with no loss of generality, we replace $x$ by $x+\pi / 2$ in (17) to obtain the following equivalent system:

$$
\begin{aligned}
& \dot{x}=\quad \sin z+\epsilon \cos y, \\
& \dot{y}=\quad \beta \cos x+\cos z, \\
& \dot{z}=-\beta \sin x+\epsilon \sin y .
\end{aligned}
$$

We will prove Theorem (5.2) and several other results that are formulated below.

Note that the unperturbed system obtained from (23) contains the Hamiltonian subsystem

$$
\dot{x}=\sin z, \quad \dot{z}=-\beta \sin x
$$

with energy

$$
h:=H(x, z)=\cos z+\beta \cos x-(1+\beta) .
$$

A typical phase portrait for (24) is depicted in Figure 1. In fact, for each $\beta$, the point $(x, z)=(0,0)$, with zero energy, is a center surrounded by a period annulus $\Omega$ consisting of periodic solutions. The outer boundary of this period annulus is a separatrix cycle with energy $-2 \beta$. A periodic solution in the period annulus has energy in the interval $-2 \beta<h<0$. Also, the system is symmetric with respect to both the $x$ axis and the $z$ axis.

The unperturbed system obtained from (23) with $\epsilon=0$ has a hyperbolic periodic solution corresponding to the hyperbolic saddle of $(24)$ at $(x, z)=(\pi, 0)$. Of course, this hyperbolic periodic solution persists. Thus, as mentioned in the introduction, using the results [10], [11], the perturbed system is a hydrodynamically unstable solution of the Euler equations. In the analysis to follow, we will give a more complete description of the hyperbolic periodic solutions that are obtained by perturbation.

Consider a periodic solution in the period annulus with energy $h$ and note that the portion of this periodic orbit in the first quadrant of the $x z$-plane is given as the graph of a function $x \mapsto z(x, h)$. Using the energy relation, we 
find

$$
\sin (z(x, h))=\left(1-(h+1+\beta-\beta \cos x)^{2}\right)^{1 / 2} .
$$

The periodic orbit crosses the positive $x$ axis at the point $x=\arccos (1+h / \beta)$ and its period is given by

$$
\begin{aligned}
\mathscr{T}(h) & =4 \int_{0}^{\arccos (1+h / \beta)} \frac{1}{\sin (z(x, h))} d x \\
& =4 \int_{0}^{\arccos (1+h / \beta)} \frac{1}{\left(1-(h+1+\beta-\beta \cos x)^{2}\right)^{1 / 2}} d x .
\end{aligned}
$$

The period can be expressed as a complete elliptic integral of the first kind. In fact, with the notation in [3] represented here in boldface, the substitution $t:=-\cos x$ in (26) results in an integral with an algebraic integrand of the form given in $[3,255.00]$. Using the Jacobi elliptic sine function $\mathrm{sn}$, a second substitution of the form

$$
\operatorname{sn}^{2} u=\frac{(\mathbf{a}-\mathbf{c})(\mathbf{b}-\mathbf{t})}{(\mathbf{b}-\mathbf{c})(\mathbf{a}-\mathbf{t})}
$$

where

$$
\mathbf{a}:=1, \quad \mathbf{b}:=-(h+\beta) / \beta, \quad \mathbf{c}:=-1, \quad \mathbf{d}:=-(2+h+\beta) / \beta
$$

and with elliptic modulus

$$
\mathbf{k}^{2}:=\frac{(\mathbf{b}-\mathbf{c})(\mathbf{a}-\mathbf{d})}{(\mathbf{a}-\mathbf{c})(\mathbf{b}-\mathbf{d})}=-h \frac{2+2 \beta+h}{4 \beta}
$$

results in the representation

$$
\mathscr{T}(h)=\frac{4}{\sqrt{\beta}} \int_{0}^{\pi / 2} \frac{d \theta}{\sqrt{1-\mathbf{k}^{2} \sin ^{2} \theta}} .
$$

Or, using the standard notation $K:=K(\mathbf{k})$ for the complete elliptic integral of the first kind with modulus $\mathbf{k}$, we have $\mathscr{T}(h)=4 K(\mathbf{k}) / \sqrt{\beta}$.

Since $\mathbf{k}$ is a decreasing function of the energy and since the complete elliptic integral of the first kind is an increasing function of $k^{2}$, the period is a monotone decreasing function of the energy. Actually, the period is $2 \pi / \sqrt{\beta}$ at the center and it increases without bound as the energy approaches $-2 \beta$.

A Poincaré section for the unperturbed flow is given by

$$
\mathscr{S}:=\left\{(x, y, z) \in \mathbb{T}^{3}: y=0 \text { and }(x, z) \in \Omega\right\} .
$$

In fact, note that for a solution starting on $\mathscr{S}$ with energy $h$ relative to (24), the $y$ component is given by $\dot{y}=h+1+\beta \neq 0$ and the solution returns to $\mathscr{S}$ when $t=2 \pi /(h+1+\beta)$.

The resonant tori correspond to periodic solutions of (24) with energy $h$ such that for some relatively prime positive integers $M$ and $N$ the following 
identity holds

$$
N \mathscr{g}(h)=M \frac{2 \pi}{1+\beta+h}
$$

Also, near a fixed resonant torus, there is, for sufficiently small $\epsilon$, a Poincaré section for the perturbed system given by an open subset $\Sigma \subset \mathscr{S}$.

To use Theorem (4.3), we must compute the fundamental matrix $\Phi(t)$ of the first variational equation of (23) in block matrix form with respect to a normal splitting adapted to $\Sigma$. For this, we let $t \mapsto(x(t), y(t), z(t))$ denote a solution of the unperturbed system with initial value on $\Sigma$, note that the linearized equations are given by

$$
\dot{\mathbf{W}}=\left(\begin{array}{llr}
0 & 0 & \cos z(t) \\
-\beta \sin x(t) & 0 & -\sin z(t) \\
-\beta \cos x(t) & 0 & 0
\end{array}\right) \mathbf{W}
$$

and define the adapted normal splitting as the sum of line bundles $\mathscr{E} \oplus \mathscr{E}^{\tan } \oplus$ $\mathscr{E}^{\text {nor }}$ generated, respectively, by the vectors

$$
\left(\begin{array}{c}
\sin z \\
\beta \cos x+\cos z \\
-\beta \sin x
\end{array}\right), \quad\left(\begin{array}{c}
\sin z \\
0 \\
-\beta \sin x
\end{array}\right), \quad\left(\begin{array}{c}
\beta \sin x \\
0 \\
\sin z
\end{array}\right)
$$

We must compute the components $a(t)$ and $b(t)$ as in the block matrix (10). To do this, we take advantage of the the fact that the unperturbed system decouples. In fact, there are functions $t \mapsto \widehat{a}(t)$ and $t \mapsto \widehat{b}(t)$ such that the principal fundamental matrix at $t=0$ for the linearization of the subsystem (24) has the block matrix form relative to $\mathscr{E}^{\text {tan }} \oplus \mathscr{E}^{\text {nor }}$ given by $\left(\begin{array}{ll}1 & \widehat{a}(t) \\ 0 & \widehat{b}(t)\end{array}\right)$. But, using this solution, we have two solutions of the full linearized system: The solution with initial value $(\sin z(0), 0,-\beta \sin x(0))$ is given by $t \mapsto(\sin z(t), 0,-\beta \sin x(t))$ while the solution with initial value $(\beta \sin x(0), 0, \sin z(0))$ is given by

$$
\left(\begin{array}{c}
0 \\
-\int_{0}^{t} \widehat{b}(s)\left(\beta^{2} \sin ^{2} x(s)+\sin ^{2} z(s)\right) d s \\
0
\end{array}\right)+\widehat{a}(t)\left(\begin{array}{c}
\sin z(t) \\
0 \\
-\beta \sin x(t)
\end{array}\right)+\widehat{b}(t)\left(\begin{array}{c}
\beta \sin x(t) \\
0 \\
\sin z(t)
\end{array}\right) .
$$

These solutions are obtained in the obvious way by substituting the solution of the subsystem (24) into the second component equation of the full linearized system. If the second solution is decomposed relative to the generators of the adapted normal splitting, we find

$$
a(t)=\widehat{a}(t)-\frac{1}{h+1+\beta} \int_{0}^{t} \widehat{b}(s)\left(\beta^{2} \sin ^{2} x(s)+\sin ^{2} z(s)\right) d s, \quad b(t)=\widehat{b}(t) .
$$

To compute $\widehat{a}$ and $\widehat{b}$ we use Diliberto's Theorem [8]. To state this theorem, let $g: \mathbb{R}^{2} \rightarrow \mathbb{R}^{2}$ be a function with components $\left(g_{1}, g_{2}\right)$, let $g^{\perp}:=J g$ where 
$J$ is the rotation matrix $\left(\begin{array}{cc}0 & -1 \\ 1 & 0\end{array}\right)$ and define

$$
\begin{aligned}
\kappa(x, z) & :=\|g(x, z)\|^{-3}\left\langle g^{\perp}(x, z), \mathrm{D} g(x, z) g\right\rangle, \\
\operatorname{div} f & :=\frac{\partial g_{1}}{\partial x}+\frac{\partial g_{2}}{\partial z} \\
\operatorname{curl} f & :=\frac{\partial g_{2}}{\partial x}-\frac{\partial g_{1}}{\partial z} .
\end{aligned}
$$

Here, $\kappa$ defines the signed scalar curvature.

Theorem 5.3 (Diliberto's Theorem). Suppose $t \mapsto \varphi_{t}$ is the flow of the differential equation $\dot{u}=g(u), u \in \mathbb{R}^{2}$. If $g(\zeta) \neq 0$, then the principal fundamental matrix solution $t \mapsto \Psi(t)$ at $t=0$ of the first variational equation

$$
\dot{\mathbf{W}}=\mathrm{D} g\left(\varphi_{t}(\zeta)\right) \mathbf{W}
$$

is such that

$$
\begin{aligned}
\Psi(t) g(\zeta) & =g\left(\varphi_{t}(\zeta)\right) \\
\Psi(t) g^{\perp}(\zeta) & =\widehat{a}(t, \zeta) g\left(\varphi_{t}(\zeta)\right)+\widehat{b}(t, \zeta) g^{\perp}\left(\varphi_{t}(\zeta)\right)
\end{aligned}
$$

where

$$
\begin{aligned}
& \widehat{b}(t, \zeta)=\frac{\|g(\zeta)\|^{2}}{\left\|g\left(\varphi_{t}(\zeta)\right)\right\|^{2}} e^{\int_{0}^{t} \operatorname{div} g\left(\varphi_{t}(\zeta)\right) d s}, \\
& \widehat{a}(t, \zeta)=\int_{0}^{t}\left(2 \kappa\left(\varphi_{s}(\zeta)\right)\left\|g\left(\varphi_{s}(\zeta)\right)\right\|-\operatorname{curl} g\left(\varphi_{s}(\zeta)\right)\right) \hat{b}(s, \zeta) d s .
\end{aligned}
$$

Proof. A proof of the theorem, albeit with a different normalization of $g^{\perp}$, is in [5].

Here, we take $g(x, z)=(\sin z, \beta \sin x)$ and note that the divergence of $g$ vanishes. Thus, we have

$$
\widehat{b}(t)=\frac{\beta^{2} \sin ^{2} x(0)+\sin ^{2} z(0)}{\beta^{2} \sin ^{2} x(t)+\sin ^{2} z(t)} .
$$

This gives

$$
a(t)=\widehat{a}(t)-\frac{\beta^{2} \sin ^{2} x(0)+\sin ^{2} z(0)}{h+1+\beta} t, \quad b(t)=\frac{\beta^{2} \sin ^{2} x(0)+\sin ^{2} z(0)}{\beta^{2} \sin ^{2} x(t)+\sin ^{2} z(t)} .
$$

Since the derivative of the unperturbed Poincare map is

$$
\left(\begin{array}{ll}
1 & a(N \mathscr{T}(h)) \\
0 & b(N \mathscr{T}(h))
\end{array}\right)=\left(\begin{array}{cc}
1 & a(N \mathscr{T}(h)) \\
0 & 1
\end{array}\right),
$$

it is clear that the resonant torus with energy $h$ is normally nondegenerate provided $a(N \mathscr{G}(h)) \neq 0$. In fact, we will show $a(N \mathscr{T}(h))<0$. For this, it suffices to show $\widehat{a}(N \mathscr{T}(h))<0$.

Let $\phi_{t}$ denote the flow of the subsystem (24) and let $\zeta$ denote a point on a periodic solution with energy $h$. With $g^{\perp}$ defined as above, let $s \mapsto \gamma(s)$ denote a solution of the orthogonal system $\dot{u}=g^{\perp}(u)$ with initial value $\gamma(0)=$ 
$\zeta$. Note that the image of $\gamma$ defines a Poincare section in the plane for the flow of (24). We let $\rho$ denote the corresponding Poincare map and compute

$$
\begin{aligned}
& \left.\frac{d}{d s} \rho^{N}(\gamma(s))\right|_{s=0}=\left.g(\zeta) \frac{d}{d s} N \mathscr{T}(H(\gamma(s)))\right|_{s=0}+\mathrm{D} \phi_{N \mathscr{G}(h)}(\zeta) g^{\perp}(\zeta) \\
& \quad=\left.g(\zeta) \frac{d}{d s} N \mathscr{T}(H(\gamma(s)))\right|_{s=0}+\widehat{a}(N \mathscr{T}(h)) g(\zeta)+\widehat{b}(N \mathscr{T}(h)) g^{\perp}(\zeta) .
\end{aligned}
$$

Since, the derivative of $s \rightarrow \rho^{N}(\gamma(s))$ is tangent to the Poincare section, it follows that the derivative of this Poincare map is $\widehat{b}(N \mathscr{T}(h))$ while $\widehat{a}(N \mathscr{T}(h))$ is the negative of $N$ times the derivative of the period function.

Recall that the derivative of the Poincare map of our planar system is the characteristic multiplier of the periodic orbit through $\zeta$. Since, in the present case, the periodic orbit lies in a period annulus we have $\rho(\gamma(s))=\gamma(s)$. In particular, this implies the derivative of the Poincare map is the identity in agreement with the fact that $\hat{b}(N \mathscr{T}(h))=1$. More importantly, we have

$$
\widehat{a}(N \mathscr{T}(h))=-\left.\frac{d}{d s} N \mathscr{T}(H(\gamma(s)))\right|_{s=0} .
$$

By checking orientations and by using the fact that $\mathscr{T}$ is a monotone decreasing function of energy, it is easy to see that

$$
\left.\frac{d}{d s} N \mathscr{T}(H(\gamma(s)))\right|_{s=0}>0 .
$$

This proves the resonant tori are normally nondegenerate.

To compute $\mathscr{M}$ we note that the perturbation is given by $(\cos y, 0, \sin y)$. A simple computation shows the $\mathscr{E}^{\text {nor }}$ component of the perturbation is

$$
\frac{\beta \sin x \cos y+\sin y \sin z}{\beta^{2} \sin ^{2} x(t)+\sin ^{2} z(t)} \text {. }
$$

Thus, ignoring the generator of $\mathscr{E}^{\text {nor }}$, by a slight abuse of notation, we have

$$
\begin{aligned}
\mathscr{M}(x(0), z(0))= & \frac{1}{\beta^{2} \sin ^{2} x(0)+\sin ^{2} z(0)} \\
& \times \int_{0}^{N \mathscr{G}(h)} \beta \sin x(t) \cos y(t)+\sin y(t) \sin z(t) d t
\end{aligned}
$$

We will find the simple zeros of $\mathscr{M}$ restricted to, $\mathscr{Z}$, the intersection of the resonant torus with $\Sigma$. For this, it is convenient to work with a different form of the integral representation of $\mathscr{M}$. To obtain it, first use the resonance relation and integration by parts to compute

$$
\int_{0}^{N \mathscr{G}(h)} \sin z(t)(\sin y(t) d t)=\frac{1}{h+1+\beta} \int_{0}^{N \mathscr{T}(h)}(\cos y(t) \cos z(t)) \dot{z}(t) d t .
$$

Next, using the energy relation,

$$
\cos z=h+1+\beta-\beta \cos x,
$$


and the last equation, we obtain

$$
\begin{aligned}
\int_{0}^{N \mathscr{T}(h)} \sin y(t) \sin z(t) d t= & -\int_{0}^{N \mathscr{T}(h)} \beta \sin x(t) \cos y(t) d t \\
& +\frac{\beta^{2}}{h+1+\beta} \int_{0}^{N \mathscr{T}(h)} \sin x(t) \cos x(t) \cos y(t) d t .
\end{aligned}
$$

Hence, it suffices to find the simple zeros of

$$
\begin{aligned}
\mathscr{M}^{*}: & =\frac{\left(\beta^{2} \sin ^{2} x(0)+\sin ^{2} z(0)\right)(h+1+\beta)}{\beta^{2}} \mathscr{M} \\
& =\int_{0}^{N \mathscr{T}(h)} \sin x(t) \cos x(t) \cos y(t) d t
\end{aligned}
$$

as the initial point varies along the resonant unperturbed periodic orbit of (24).

To evaluate the integral representation of $\mathscr{M}^{*}$, we will express the function $t \mapsto \sin x(t) \cos x(t)$ in terms of Jacobian elliptic functions. For this, we let $t \mapsto(x(t), y(t))$ denote the solution of (24) with initial value $\left(0, \cos ^{-1}(1+h)\right)$, the intersection point of $\mathscr{Z}$ with the positive $z$ axis. Also, we introduce a local coordinate $\theta, 0 \leq \theta<4 K$, on $\mathscr{Z}$ by $\theta \mapsto(x(\theta / \sqrt{\beta}), z(\theta / \sqrt{\beta}))$. In particular, we have

$$
\mathscr{M}^{*}(\theta)=\int_{0}^{N \mathscr{T}(h)} \sin x(t+\theta \sqrt{\beta}) \cos x(t+\theta \sqrt{\beta}) \cos y(t) d t
$$

Since $\dot{x}(\tau) / \sin z(\tau)=1$, we have

$$
\tau=\int_{0}^{\tau} \frac{\dot{x}(s)}{\sin z(s)} d s .
$$

With $\sigma=x(s)$ on the domain $0 \leq s \leq 2 K / \sqrt{\beta}$ and in view of the energy relation (25), the last equation can be recast in the form

$$
\tau=\int_{0}^{x(\tau)} \frac{1}{\sqrt{1-(h+1+\beta-\beta \cos \sigma)^{2}}} d \sigma .
$$

Moreover, with $\mathbf{t}=-\cos \sigma$ and with $\mathbf{a}, \mathbf{b}, \mathbf{c}, \mathbf{d}$ as in (28),

$$
\tau=\frac{1}{\beta} \int_{-1}^{-\cos x(\tau)} \frac{1}{\sqrt{(\mathbf{a}-\mathbf{t})(\mathbf{b}-\mathbf{t})(\mathbf{t}-\mathbf{c})(\mathbf{t}-\mathbf{d})}} d \mathbf{t} .
$$

We define $L$ by the equation

$$
\operatorname{sn}^{2} L=\frac{(\mathbf{a}-\mathbf{c})(\mathbf{b}+\cos x(\tau))}{(\mathbf{b}-\mathbf{c})(\mathbf{a}+\cos x(\tau))}
$$

and use the change of variables (27) to obtain

$$
\tau=-\frac{2}{\beta \sqrt{(\mathbf{a}-\mathbf{c})(\mathbf{b}-\mathbf{d})}} \int_{K}^{L} d u=\frac{1}{\beta}(K-L) .
$$


Then, solving for $\cos x(\tau)$, we find

$$
\cos x(t)=\frac{2 h+2 \beta-h \operatorname{sn}^{2}(K-t \sqrt{\beta})}{2 \beta+h \operatorname{sn}^{2}(K-t \sqrt{\beta})} .
$$

For $2 K / \sqrt{\beta} \leq t \leq 4 K / \sqrt{\beta}$, it is easy to see $\cos x(t)$ is given by the same formula except the argument of sn is $K+t \sqrt{\beta}$. Since $\operatorname{sn}(K+u)=$ $\operatorname{sn}(K-u)$, the above formula is actually valid for all $t \in \mathbb{R}$. In addition, using the trigonometric identity $\sin ^{2} u=1-\cos ^{2} u$ and taking account of the sign, we find

$$
\sin x(t)=2 \sqrt{-h(h+2 \beta)} \frac{\operatorname{cn}(K-t \sqrt{\beta})}{2 \beta+h \operatorname{sn}^{2}(K-t \sqrt{\beta})} .
$$

Define

$$
\operatorname{vn} u:=\operatorname{vn}(u, \mathbf{k}):=2 \sqrt{-h(h+2 \beta)} \operatorname{cn} u \frac{2 h+2 \beta-h \mathrm{sn}^{2} u}{\left(2 \beta+h \mathrm{sn}^{2} u\right)^{2}}
$$

and note that

$$
\mathscr{M}^{*}(\theta)=\int_{0}^{4 N K / \sqrt{\beta}} \operatorname{vn}(K-t \sqrt{\beta}-\theta) \cos ((h+1+\beta) t) d t .
$$

We will use the properties of the elliptic function vn inherited from the periodicity properties of the Jacobian elliptic functions sn and $\mathrm{cn}$, see [3, pp. 18-28], to analyze $\mathscr{M}^{*}$. In particular, we note here that

$$
\operatorname{vn}(-u)=\operatorname{vn}(u), \quad \operatorname{vn}(u+4 K)=\operatorname{vn}(u) .
$$

Set $s=\theta+t \sqrt{\beta}-K$ and compute

$$
\begin{aligned}
\sqrt{\beta} \cdot \mathscr{K}^{*}(\theta) & =\cos \left(\frac{h+1+\beta}{\sqrt{\beta}}(K-\theta)\right) \int_{\theta-K}^{\theta-K+4 N K} \operatorname{vn} s \cos \left(\frac{h+1+\beta}{\sqrt{\beta}} s\right) d s \\
& -\frac{1}{\sqrt{\beta}} \sin \left(\frac{h+1+\beta}{\sqrt{\beta}}(K-\theta)\right) \int_{\theta-K}^{\theta-K+4 N K} \operatorname{vn} s \sin \left(\frac{h+1+\beta}{\sqrt{\beta}} s\right) d s .
\end{aligned}
$$

Also, note that the resonance relation can be recast as the equality

$$
\frac{h+1+\beta}{\sqrt{\beta}}=\frac{M}{N}\left(\frac{\pi}{2 K}\right) .
$$

In particular, each integrand is periodic with period $4 N K$. Thus, both integrals are unchanged when the interval of integration is replaced by $-2 N K \leq s \leq$ $2 N K$. Moreover, taking into account the fact that vn is an even function, it follows that the second integral vanishes. Hence, using the new variable $\sigma=\pi s /(2 N K)$, we compute

$$
\begin{aligned}
\sqrt{\beta} \mathscr{M}^{*}(\theta) & =\cos \left(\frac{M}{N}\left(\frac{\pi}{2 K}\right)(K-\theta)\right) \int_{-2 N K}^{2 N K} \operatorname{vn} s \cos \left(\frac{M}{N}\left(\frac{\pi}{2 K}\right) s\right) d s \\
& =N \frac{2 K}{\pi} \cos \left(\frac{M}{N}\left(\frac{\pi}{2 K}\right)(K-\theta)\right) \int_{-\pi}^{\pi} \operatorname{vn}\left(N \frac{2 K}{\pi} \sigma\right) \cos (M \sigma) d s
\end{aligned}
$$


Since $\sigma \mapsto \operatorname{vn}(2 K \sigma / \pi)$ is an even nonconstant nonelementary $2 \pi$ periodic function, it is represented by a Fourier cosine series with infinitely many nonzero Fourier coefficients. This observation is all that is required below to prove the existence of any preassigned number of hyperbolic periodic orbits after perturbation. However, we will prove more.

Proposition 5.4. The Fourier series representation of the function

$$
\sigma \mapsto \operatorname{vn}(2 K \sigma / \pi, \mathbf{k}),
$$

defined by (38) with $\mathbf{k}$ given by (29), has the form

$$
\operatorname{vn}\left(\frac{2 K}{\pi} \sigma\right)=v_{0}+\sum_{j=1}^{\infty} v_{2 j+1} \cos ((2 j+1) \sigma) \text {. }
$$

Moreover, $v_{2 j+1} \neq 0$ for infinitely many integers $j \geq 0$.

Assuming the validity of the proposition, it follows from (39) that the bifurcation function, defined on the intersection of the $(M: N)$ resonant torus with the Poincare section, vanishes identically unless there is a nonnegative integer $n$ such that $v_{2 n+1} \neq 0$ and $M=(2 n+1) N$. If this is the case, then since $M$ and $N$ are relatively prime, we must have $N=1, M=2 n+1$ and

$$
\begin{aligned}
\mathscr{M}^{*}(\theta) & =\frac{2 K}{\pi} \frac{1}{\sqrt{\beta}} \cos \left(\frac{\pi}{2 K}(2 n+1)(K-\theta)\right) \pi v_{2 n+1} \\
& =-\frac{2 K}{\sqrt{\beta}} v_{2 n+1} \sin \left((2 n+1) \frac{\pi}{2}\right) \sin \left((2 n+1) \frac{\pi}{2 K} \theta\right) .
\end{aligned}
$$

Thus, $\mathscr{M}$ has $2(2 n+1)$ simple zeros.

Here, we must show that infinitely many $(2 n+1: 1)$ resonant tori actually exist. For this, recall that the period function $h \mapsto \mathscr{T}(h)$ is a monotone decreasing function on the interval $-2 \beta<h \leq 0$ with $\mathscr{T}(0)=2 \pi / \sqrt{\beta}$ and $\lim _{h \rightarrow-2 \beta^{+}} \mathscr{T}(h)=\infty$. Also, the resonance relation takes the form

$$
\mathscr{T}(h)=(2 n+1) \frac{2 \pi}{1+\beta+h} \text {. }
$$

By comparison of the graphs of the left and right sides of this equation as functions of $h$, it is easy to see that for fixed $\beta$, in the range $0<\beta \leq 1$, and for $n \geq 0$, there is an $h$ satisfying the resonance equation provided

$$
\frac{2 \pi}{\sqrt{\beta}}<(2 n+1) \frac{2 \pi}{1+\beta} .
$$

Thus we have the following fact: For fixed $\beta$ in the range $0<\beta \leq 1$, there is $a(2 n+1: 1)$ resonant torus provided

$$
2 n+1>\frac{1+\beta}{\sqrt{\beta}} .
$$

In particular, for each such $\beta$ there are infinitely many resonant tori.

We cannot conclude from the results presented here (without proving an additional uniformity estimate) that there are infinitely many periodic orbits after perturbation. In effect, for each resonant torus we have only proved there is a 
sufficiently small $\epsilon$ for which the corresponding simple zeros of the bifurcation function persist, but the necessary $\epsilon$ may shrink to zero as the resonant tori approach the boundary of the period annulus. The fact that there are infinitely many periodic solutions after perturbation will be proved in Section 5.3.

We will show $2 n+1$ of the zeros of $\mathscr{M}$ on the $(2 n+1: 1)$ resonant torus correspond to perturbed families of hyperbolic saddle orbits. For this, we proceed as in the discussion following equation (21). As before, the derivative of the parametrized Poincare map is given by

$$
\mathrm{D} \mathscr{P}^{N}(x, z, \epsilon)=\mathrm{D} p^{N}(x, z)+\epsilon \mathrm{D} \mathscr{P}_{\epsilon}^{N}(x, z, 0)+O\left(\epsilon^{2}\right) .
$$

It suffices to compute the eigenvalues of this derivative at the unperturbed persistent periodic orbit that we will assume corresponds to the parameter value $\theta_{0}$. This computation is carried out using the normal coordinates.

In view of (35), we have

$$
\mathrm{D} p^{N}\left(\theta_{0}\right)=\left(\begin{array}{cc}
1 & a(N \mathscr{T}(h)) \\
0 & 1
\end{array}\right)
$$

with $a(N \mathscr{T}(h))<0$. Also, in view of $(14)$, and the fact that $b(N \mathscr{T}(h))=1$, we have

$$
\left(\mathscr{P}_{\epsilon}^{N}\right)^{\text {nor }}=\mathscr{M}(\theta) .
$$

Thus,

$$
\mathrm{D} \mathscr{P}^{N}(x, z, \epsilon)=\left(\begin{array}{cc}
1 & a(N \mathscr{T}(h)) \\
0 & 1
\end{array}\right)+\epsilon\left(\begin{array}{cc}
p_{11}(\theta) & p_{12}(\theta) \\
\mathscr{M}^{\prime}(\theta) & p_{22}(\theta)
\end{array}\right)+O\left(\epsilon^{2}\right)
$$

where the $p_{i j}$ can be computed, if desired, from (14).

The eigenvalues of $\mathrm{D}^{N}(x, z, \epsilon)$ are given by

$$
1 \pm \sqrt{\epsilon} \sqrt{a(N \mathscr{T}(h)) \mathscr{K}^{\prime}\left(\theta_{0}\right)}+O(\epsilon) .
$$

Since $a(N \mathscr{T}(h))<0$, the family of perturbed orbits branching from the persistent unperturbed periodic orbit corresponding to $\theta_{0}$ will consist of hyperbolic saddle orbits provided $\mathscr{M}^{\prime}\left(\theta_{0}\right)<0$. This condition is satisfied for $2 n+1$ of the zeros of $\mathscr{K}^{*}(\theta)$, as required.

The remaining $2 n+1$ perturbed periodic orbits are elliptic. This follows from the fact that the Poincare map is area preserving. In fact, the $A B C$ vector field is a contact vector field, [4]. In particular, its flow preserves the volume $\lambda \wedge d \lambda$ on $\mathbb{T}^{3}$ given by the contact form $\lambda$. This fact can be used to show that our Poincare map is area preserving.

To determine exactly which resonant tori are excited by the perturbation, we must determine which Fourier coefficients $v_{2 j+1}$ are nonzero. For this, we have the following proposition.

Proposition 5.5. Suppose the $(M: N)$ resonant torus has energy $h$ in the range $-2 \beta<h<0$. If $0<r^{*}<\pi / 2$ is the unique solution of the equation

(40) $\operatorname{sn}\left(\frac{2 K}{\pi} r^{*}\right)=\left(\frac{2}{2+2 \beta+h}\right)^{1 / 2}=\left(\frac{2}{\beta+1+\left((\beta+1)^{2}-4 \beta \mathbf{k}^{2}\right)^{1 / 2}}\right)^{1 / 2}$, 
then $v_{2 j+1} \neq 0$ if and only if

$$
(2 j+1) \sin \left((2 j+1) r^{*}\right)-\frac{M}{N} \cos \left((2 j+1) r^{*}\right) \neq 0 .
$$

In particular, for the $(2 n+1: 1)$ resonance, that is, for the elliptic modulus $0<\mathbf{k}<1$ such that

$$
K(\mathbf{k})=(2 n+1) \frac{\pi}{2}\left(\frac{\beta}{(\beta+1)^{2}-4 \beta \mathbf{k}^{2}}\right),
$$

the Fourier coefficient $v_{2 n+1}$ is not zero if and only if $(2 n+1) r^{*}$ is not equivalent to $\pi / 4$ modulo $\pi$.

Numerical experiments suggest that, in fact, for fixed $\beta$ in the range $0<$ $\beta<1$, there is at most one integer $n$, in the range $n=1,2 \ldots, \infty$, such that the $(2 n+1: 1)$ resonant torus is not excited, that is, such that $v_{2 n+1}=0$. Also, these experiments suggest that such exceptional resonant tori actually exist. For example, it appears that there is a choice of $\beta$, in the range $5 / 20<\beta<7 / 20$, such that the $(3: 1)$ resonant torus is not excited. More precisely, for this $\beta$ there is a $\mathbf{k}$ such that

$$
\begin{aligned}
K(\mathbf{k}) & =3 \frac{\pi}{2}\left(\frac{\beta}{(\beta+1)^{2}-4 \beta \mathbf{k}^{2}}\right), \\
\operatorname{sn}\left(\frac{2 K}{\pi}\left(\frac{1}{3}\left(\frac{\pi}{4}+\pi\right)\right)\right) & =\left(\frac{2}{\beta+1+\left((\beta+1)^{2}-4 \beta \mathbf{k}^{2}\right)^{1 / 2}}\right)^{1 / 2} .
\end{aligned}
$$

It remains to prove Proposition (5.4) and Proposition (5.5).

Proof. Let $K^{\prime}$ denote the complete elliptic integral complementary to $K$, define $\tau:=i K^{\prime} / K$ and, for notational convenience, define $\operatorname{mn}(x):=\operatorname{vn}(2 K x / \pi)$. We will compute

$$
I_{m}:=\int_{-\pi}^{\pi} \operatorname{rn}(x) e^{i m x} d x, \quad m \neq 0,
$$

by a contour integration of the function $G(z):=\operatorname{mn}(z) e^{i m x}$ around the parallelogram, $\mathscr{G}$, in the complex plane with vertices $-\pi, \pi, \pi \tau$ and $\pi \tau-2 \pi$, cf. [16].

The following properties of the function $\mathrm{rn}$ are easy to check using the double periodicity of sn and $\mathrm{cn}$ :

$$
\begin{aligned}
\mathrm{m}(-x) & =\mathrm{rn}(x), & \mathrm{rn}(x \pm \pi) & =-\operatorname{mn}(x), \\
\mathrm{mn}(x \pm 2 \pi) & =\mathrm{rn}(x), & \operatorname{mn}(x \pm \pi \tau) & =-\operatorname{mn}(x) .
\end{aligned}
$$

The sum of the path integrals over the oriented edges $[\pi, \pi \tau]$ and $[\pi \tau-$ $2 \pi,-\pi]$ of $\mathscr{G}$ is zero. To see this, let $t \mapsto \gamma(t)$ denote a parametrization of the edge $[\pi, \pi \tau]$, for example; let $\gamma(t)=(1-t) \pi+t \pi \tau$. We have

$$
\int_{[\pi, \pi \tau]} G(z) d z=\int_{0}^{1} \operatorname{mn}(\gamma(t)) e^{i m \gamma(t)} \dot{\gamma}(t) d t .
$$

Clearly, $t \mapsto \gamma(t)-2 \pi$ parametrizes the edge $[\pi \tau-2 \pi,-\pi]$. Thus, we have

$$
\int_{[\pi \tau-2 \pi,-\pi]} G(z) d z=-\int_{0}^{1} \mathrm{mn}(\gamma(t)-2 \pi) e^{i m \gamma(t)} \dot{\gamma}(t) d t
$$


But, since $\operatorname{mn}(x-2 \pi)=\operatorname{mn}(x)$, we obtain the desired result.

Consider the integral

$$
I_{m}^{*}:=\int_{[\pi \tau, \pi \tau-2 \pi]} \operatorname{mn}(z) e^{i m z} d z .
$$

If $t \mapsto \gamma(t)$ parametrizes $[-\pi, \pi]$, on the interval $0 \leq t \leq 1$, then $\gamma(t)-\pi+\pi \tau$ parametrizes $[\pi \tau-2 \pi, \pi \tau]$. Thus,

$$
\begin{aligned}
I_{m}^{*} & =-\int_{0}^{1} \operatorname{mn}(\gamma(t)-\pi+\pi \tau) e^{i m \gamma(t)} e^{-i m \pi} e^{i m \pi \tau} \dot{\gamma}(t) d t \\
& =-e^{-i m \pi} e^{i m \pi \tau} I_{m} .
\end{aligned}
$$

In particular, we have now proved

$$
\int_{\mathscr{G}} G(z) d z=I_{m}+I_{m}^{*}=\left(1-e^{-i m \pi} e^{i m \pi \tau}\right) I_{m} .
$$

We will soon see that $G$ is a meromorphic function that is analytic on $\mathscr{G}$. If we let $\operatorname{Res}(v):=\operatorname{Res}(G, v)$ denote the residue of $G$ at the pole $z=v$, then, by the Residue Theorem,

$$
I_{m}=2 \pi i\left(1-e^{-i m \pi} e^{i m \pi \tau}\right)^{-1} \sum \operatorname{Res}(G, v)
$$

where the sum is over all poles enclosed by $\mathscr{G}$.

To determine the poles of $G$ we must consider the poles of the functions sn and $\mathrm{cn}$ together with the zeros of the denominator of $\mathrm{rn}$, that is, the zeros of the function defined by $g(z):=2 \beta+h \operatorname{sn}^{2}(2 K z / \pi)$. Using the fact that sn $u$ has a simple pole at the points $i K^{\prime}$ and $2 K+i K^{\prime}$ modulo the lattice generated by $\left(4 K, 2 i K^{\prime}\right)$ and cn $u$ has a simple pole at $i K^{\prime}$ and $2 K+i K^{\prime}$ modulo $\left(4 K, 2 K+2 i K^{\prime}\right)$, it is easy to see the corresponding points enclosed by $\mathscr{G}$ are $\pi \tau / 2$ and $\pi \tau / 2-\pi$. However, since $\operatorname{sn}(2 K z / \pi)$ and $\operatorname{cn}(2 K z / \pi)$ both have simple poles at these point, the leading term of the Laurent series of the numerator of $\mathrm{rn}$ at each pole has order 3 while the denominator has a pole of order 4 . In other words, the points $\pi \tau / 2$ and $\pi \tau / 2-\pi$ are removable singularities of $G$ and the only poles are the zeros of $g$.

Recall that $-2 \beta<h<0$ and consider the function $g$. Its zeros are the roots of the equations

$$
\operatorname{sn}\left(\frac{2 K}{\pi} z\right)= \pm \sqrt{\frac{2 \beta}{-h}} .
$$

Since, for real $u$, we have $|\operatorname{sn} u| \leq 1$, these equations have no real roots. To determine the complex roots, recall the addition formula for $s n,[3,125.01]$,

$$
\begin{aligned}
\operatorname{sn}(u+i v, \mathbf{k})= & \frac{\operatorname{sn}(u, \mathbf{k}) \operatorname{dn}\left(v, \mathbf{k}^{\prime}\right)}{1-\operatorname{sn}^{2}\left(v, \mathbf{k}^{\prime}\right) \operatorname{dn}^{2}(u, \mathbf{k})} \\
& +i \frac{\operatorname{cn}(u, \mathbf{k}) \operatorname{dn}(u, \mathbf{k}) \operatorname{sn}\left(v, \mathbf{k}^{\prime}\right) \operatorname{cn}\left(v, \mathbf{k}^{\prime}\right)}{1-\operatorname{sn}^{2}\left(v, \mathbf{k}^{\prime}\right) \operatorname{dn}^{2}(u, \mathbf{k})}
\end{aligned}
$$

where $\mathbf{k}^{\prime}:=\sqrt{1-\mathbf{k}^{2}}$ is the complementary elliptic modulus. 
In order to obtain a real value with a complex argument, we must have

$$
\operatorname{cn}(u, \mathbf{k}) \operatorname{dn}(u, \mathbf{k}) \operatorname{sn}\left(v, \mathbf{k}^{\prime}\right) \operatorname{cn}\left(v, \mathbf{k}^{\prime}\right)=0 .
$$

Thus, $u=K \bmod 2 K, v=0 \bmod 2 K^{\prime}$ or $v=K^{\prime} \bmod 2 K^{\prime}$. If $u=K \bmod$ $2 K$, then $\operatorname{sn}(u+i v, \mathbf{k})= \pm 1 / \operatorname{dn}\left(v, \mathbf{k}^{\prime}\right)$. For real $v$, we have $\mathbf{k} \leq \operatorname{dn}\left(v, \mathbf{k}^{\prime}\right) \leq$ 1. Thus, from (29),

$$
1 \leq \frac{1}{\operatorname{dn}\left(v, \mathbf{k}^{\prime}\right)} \leq \frac{1}{\mathbf{k}} \leq \sqrt{\frac{2 \beta}{-h}} \sqrt{\frac{2}{2+2 \beta+h}}<\sqrt{\frac{2 \beta}{-h}}
$$

and it follows that $\operatorname{sn}(u+i v, \mathbf{k}) \neq \pm \sqrt{2 \beta} / \sqrt{-h}$. If $v=0 \bmod 2 K^{\prime}$, then $\operatorname{sn}(u+i v, \mathbf{k})=\operatorname{sn} u$ and, as above, (41) has no solutions. However, if $v=$ $K^{\prime} \bmod 2 K^{\prime}$, then $\operatorname{sn}(u+i v)=1 /(\mathbf{k} \operatorname{sn}(u, \mathbf{k}))$ and $(41)$ has solutions.

We have

$$
\operatorname{sn}\left(\frac{2 K}{\pi}(x+\pi \tau / 2)\right)=\operatorname{sn}\left(\frac{2 K}{\pi} x+i K^{\prime}\right)=\left(\mathbf{k s n}\left(\frac{2 K}{\pi} x\right)\right)^{-1} .
$$

Therefore, we must solve

$$
\mathbf{k s n}\left(\frac{2 K}{\pi} x\right)= \pm \sqrt{\frac{-h}{2 \beta}} .
$$

There is a unique real number $0<r^{*}<\pi / 2$, such that

$$
\operatorname{sn}\left(\frac{2 K}{\pi} r^{*}\right)=\frac{1}{\mathbf{k}} \sqrt{\frac{-h}{2 \beta}}=\sqrt{\frac{2}{2+2 \beta+h}} .
$$

In terms of this value, we have

$$
\begin{gathered}
\operatorname{sn}\left(\frac{2 K}{\pi}\left(r^{*}-\pi\right)\right)=\operatorname{sn}\left(\frac{2 K}{\pi} r^{*}\right), \quad \operatorname{sn}\left(\frac{2 K}{\pi}\left(-r^{*}\right)\right)=-\operatorname{sn}\left(\frac{2 K}{\pi} r^{*}\right), \\
\operatorname{sn}\left(\frac{2 K}{\pi}\left(-r^{*}-\pi\right)\right)=-\operatorname{sn}\left(\frac{2 K}{\pi} r^{*}\right) .
\end{gathered}
$$

In other words, the poles of $G$ enclosed by $\mathscr{G}$ are the four points

$$
r^{*}+\frac{\pi}{2} \tau, \quad r^{*}-\pi+\frac{\pi}{2} \tau, \quad-r^{*}+\frac{\pi}{2} \tau, \quad-r^{*}-\pi-\frac{\pi}{2} \tau .
$$

We claim these poles of $G$ are simple zeros of $g$. To see this, note that, [3, 371.01],

$$
\frac{\partial}{\partial z}\left(2 \beta+h \operatorname{sn}^{2} z\right)=2 h \operatorname{sn} z \mathrm{cn} z \mathrm{dn} z .
$$

If this derivative vanishes at one of the zeros, $s$, of $2 \beta+h \mathrm{sn}^{2} z$, then

$$
\begin{gathered}
\operatorname{sn}^{2} s=\frac{2 \beta}{-h}, \quad \operatorname{cn}^{2} s=1-\operatorname{sn}^{2} s=1+\frac{2 \beta}{h}, \\
\operatorname{dn}^{2} s=1-k^{2} \operatorname{sn}^{2} s=1+k^{2} \frac{2 \beta}{h}
\end{gathered}
$$

and we must have

$$
0=\operatorname{cn}^{2} s \operatorname{dn}^{2} s=\frac{(h+2 \beta)\left(h+2 \mathbf{k}^{2} \beta\right)}{h^{2}} .
$$


But, $h+2 \beta \neq 0$ and $h+2 \mathbf{k}^{2} \beta \neq 0$. Hence, $s$ is a simple zero.

Let $r$ denote one of the poles of $G$. Since $r$ corresponds to a simple zero of $g$, we have the Taylor representation

$$
2 \beta+h \operatorname{sn}^{2}\left(\frac{2 K}{\pi} z\right)=d_{1}(z-r)+d_{2}(z-r)^{2}+O\left((z-r)^{3}\right),
$$

with $d_{1} \neq 0$. Using this representation, we obtain

$$
2 h+2 \beta-h \operatorname{sn}^{2}\left(\frac{2 K}{\pi} z\right)=2 h+4 \beta-d_{1}(z-r)+O\left((z-r)^{2}\right) .
$$

In addition, we will use the Taylor series

$$
\begin{aligned}
\operatorname{cn}^{2}\left(\frac{2 K}{\pi} z\right) & =c_{0}+c_{1}(z-r)+O\left((z-r)^{2}\right), \\
e^{i m z} & =e^{i m r}+i m e^{i m r}(z-r)+O\left((z-r)^{2}\right) .
\end{aligned}
$$

If the above Taylor series are substituted into the expression for $g$, the Laurent series at $r$ is easily computed. There is a pole of order 2 at $r$ and the residue at this pole is

$$
\begin{aligned}
\operatorname{Res}(r)= & 2 d_{1}^{-2} \sqrt{-h(h+2 \beta)} \\
& \times e^{i m r}\left((2 h+4 \beta) c_{1}-c_{0} d_{1}+(2 h+4 \beta) c_{0}\left(i m-2 d_{2} d_{1}^{-1}\right)\right) .
\end{aligned}
$$

Also, using [3, 731.02, 731.03], we compute

$$
\begin{aligned}
c_{0}(r) & =\operatorname{cn}\left(\frac{2 K}{\pi} r\right), \\
c_{1}(r) & =-\frac{2 K}{\pi} \operatorname{sn}\left(\frac{2 K}{\pi} r\right) \operatorname{dn}\left(\frac{2 K}{\pi} r\right), \\
d_{1}(r) & =2 h \frac{2 K}{\pi} \operatorname{sn}\left(\frac{2 K}{\pi} r\right) \operatorname{cn}\left(\frac{2 K}{\pi} r\right) \mathrm{dn}\left(\frac{2 K}{\pi} r\right), \\
d_{2}(r)=h \frac{2 K}{\pi}\left(\operatorname{cn}^{2}\left(\frac{2 K}{\pi} r\right) \mathrm{dn}^{2}\left(\frac{2 K}{\pi} r\right)-\operatorname{sn}^{2}\left(\frac{2 K}{\pi} r\right) \mathrm{dn}^{2}\left(\frac{2 K}{\pi} r\right)\right. & \left.\quad-\mathbf{k}^{2} \operatorname{sn}^{2}\left(\frac{2 K}{\pi} r\right) \mathrm{cn}^{2}\left(\frac{2 K}{\pi} r\right)\right),
\end{aligned}
$$

and note that

$$
\begin{array}{lll}
c_{0}(r-\pi) & =-c_{0}(r), & c_{1}(r-\pi)=-c_{1}(r), \\
d_{1}(r-\pi)=d_{1}(r), & d_{2}(r-\pi)=d_{2}(r) .
\end{array}
$$

Then, from (47), we obtain

$$
\operatorname{Res}(r)=-e^{-i m \pi} \operatorname{Res}(r-\pi) .
$$

Thus, if $m$ is an even integer, $e^{-i m \pi}=1$ and it follows immediately that the sum of the residues is zero. In this case $I_{m}=0$. This completes the proof of the first statement of Proposition (5.4).

To show there are infinitely many nonzero Fourier coefficients, suppose the contrary and note that, by what we have just proved, this would mean the function $\mathrm{rn}$, for each fixed value of the elliptic modulus, is represented by a 
trigonometric polynomial. The trigonometric polynomial is an entire function while $\mathrm{rn}$ is a meromorphic function that agrees with the trigonometric polynomial on the real line. This implies the two functions agree on the domain in the complex plane obtained by removing the poles of $\mathrm{m}$. This is a contradiction. For example, the trigonometric polynomial remains bounded in a punctured neighborhood of one of the poles while $\mathrm{m}$ does not. This completes the proof of Proposition (5.4).

Using (29) we find

$$
h=\sqrt{(\beta+1)^{2}-4 \beta \mathbf{k}^{2}}-(\beta+1) .
$$

Substitution of this expression into (42) yields equation (40).

If $m$ is odd,

$$
I_{m}=2 \pi i\left(1+e^{i m \pi \tau}\right)\left(2 \operatorname{Res}\left(r^{*}+\frac{\pi}{2} \tau\right)+2 \operatorname{Res}\left(-r^{*}+\frac{\pi}{2} \tau\right)\right)
$$

with the residues given by (47). We will prove the first statement of Proposition (5.5) by computing these residues.

As we have seen above, $\operatorname{sn}\left(u+i K^{\prime}\right)=1 /(\mathbf{k} \operatorname{sn} u)$. By similar computations using the addition formulas for $\mathrm{cn}$ and $\mathrm{dn},[3,125.01]$, we also have

$$
\operatorname{cn}\left(u+i K^{\prime}\right)=-i \frac{\operatorname{dn} u}{\mathbf{k} \operatorname{sn} u}, \quad \operatorname{dn}\left(u+i K^{\prime}\right)=-i \frac{\operatorname{cn} u}{\mathbf{k} \operatorname{sn} u} .
$$

In particular, with $\rho:=2 K r^{*} / \pi$, we compute

$$
-i C_{0}:=c_{0}\left(r^{*}+\frac{\pi}{2} \tau\right)=-i \frac{\operatorname{dn} \rho}{\mathbf{k} \operatorname{sn} \rho}=-c_{0}\left(-r^{*}+\frac{\pi}{2} \tau\right)
$$

Similarly,

$$
\begin{aligned}
i C_{1} & :=c_{1}\left(r^{*}+\frac{\pi}{2} \tau\right)=c_{1}\left(-r^{*}+\frac{\pi}{2} \tau\right), \\
D_{1} & :=d_{1}\left(r^{*}+\frac{\pi}{2} \tau\right)=-d_{1}\left(-r^{*}+\frac{\pi}{2} \tau\right), \\
D_{2} & :=d_{2}\left(r^{*}+\frac{\pi}{2} \tau\right)=d_{2}\left(-r^{*}+\frac{\pi}{2} \tau\right) .
\end{aligned}
$$

Using these identities and the definitions

$$
\begin{gathered}
L:=e^{i m \pi \tau / 2} 2 D_{1}^{-2} \sqrt{-h(h+2 \beta)}, \quad Q:=(2 h+4 \beta) m C_{0}, \\
R:=(2 h+4 \beta) C_{1}+C_{0} D_{1}+2(2 h+4 \beta) C_{0} D_{2} D_{1}^{-1},
\end{gathered}
$$

a calculation shows

$$
\begin{aligned}
\operatorname{Res}\left(r^{*}+\frac{\pi}{2} \tau\right)+\operatorname{Res}\left(-r^{*}+\frac{\pi}{2} \tau\right) & =L\left(e^{i m r^{*}}(Q+i R)+e^{-i m r^{*}}(-Q+i R)\right) \\
& =2 L i\left(Q \sin \left(m r^{*}\right)+R \cos \left(m r^{*}\right)\right) .
\end{aligned}
$$

The real number $L$ is not zero. In order to find a condition equivalent to the inequality

$$
Q \sin \left(m r^{*}\right)+R \cos \left(m r^{*}\right) \neq 0,
$$


we will determine $Q$ and $R$. Since $0<2 K r^{*} / \pi<K$, using (42), we have

$$
\begin{aligned}
& \operatorname{cn} \rho=\sqrt{1-\operatorname{sn}^{2} \rho}=\left(\frac{2 \beta+h}{2+2 \beta+h}\right)^{1 / 2}, \\
& \operatorname{dn} \rho=\sqrt{1-k^{2} \operatorname{sn}^{2} \rho}=\left(\frac{2 \beta+h}{2 \beta}\right)^{1 / 2} .
\end{aligned}
$$

By a straightforward computation using these formulas, we find

$$
\begin{aligned}
& Q=m(2 h+4 \beta)\left(\frac{2 \beta+h}{-h}\right)^{1 / 2}, \\
& R=-\frac{4 K}{\pi}\left(\frac{2 \beta+h}{-h \beta}\right)^{1 / 2}(2 \beta+h)(\beta+h+1)
\end{aligned}
$$

and, with

$$
C:=2(2 \beta+h)\left(\frac{2 \beta+h}{-h}\right)^{1 / 2},
$$

we find

$$
\begin{aligned}
Q \sin \left(m r^{*}\right)+R \cos \left(m r^{*}\right) & =C\left(m \sin \left(m r^{*}\right)-\left(\frac{2 K}{\pi}\right) \frac{\beta+h+1}{\sqrt{\beta}} \cos \left(m r^{*}\right)\right) \\
& =C\left(m \sin \left(m r^{*}\right)-\frac{M}{N} \cos \left(m r^{*}\right)\right) .
\end{aligned}
$$

This completes the proof of the first statement of Proposition (5.5).

5.3. Perturbation of heteroclinic orbits and chaos. Consider the ABC system (23) for $0<\beta<1$. The subsystem (24) has a period annulus at the origin that is bounded by two hyperbolic saddle points, $(x, z)=(0, \pm \pi)$, and two connecting heteroclinic orbits (one saddle and a homoclinic orbit if we view the system on $\mathbb{T}^{3}$ ). The boundary of the period annulus has energy $h=-2 \beta$. In fact, the boundary is a subset of the graph of

$$
\cos z+\beta \cos x=1-\beta .
$$

In this subsection we will determine the fate of the heteroclinic orbits after bifurcation by computing the "Melnikov" function.

Let $\theta$ denote the local coordinate along one of the heteroclinic orbits. The Melnikov function is given (up to a scalar multiple) by

$$
\begin{aligned}
\mathscr{M}_{\infty}^{*}(\theta) & =\int_{-\infty}^{\infty} \sin (x(t+\theta)) \cos (x(t+\theta)) \cos (y(t)) d t \\
& =\int_{-\infty}^{\infty} \sin x(t) \cos x(t) \cos ((1-\beta)(t-\theta)) d t \\
& =I_{s} \cos ((1-\beta) \theta)+I_{c} \sin ((1-\beta) \theta)
\end{aligned}
$$

where we have defined

$$
\begin{aligned}
& I_{s}:=\int_{-\infty}^{\infty} \sin x(t) \cos x(t) \sin ((1-\beta) t) d t, \\
& I_{c}:=\int_{-\infty}^{\infty} \sin x(t) \cos x(t) \cos ((1-\beta) t) d t .
\end{aligned}
$$


The derivation and geometric properties of the Melnikov function are well known, see $[15, \S 4.5]$ for a detailed account. The function $\mathscr{M}_{\infty}^{*}$ is obtained from the function $\mathscr{K}^{*}$, defined by equation (37). In fact, $\mathscr{M}_{\infty}^{*}$ is the limiting value of function $\mathscr{K}^{*}$ as the resonant unperturbed periodic solutions approach the boundary of the period annulus. However, the fact that the simple zeros of $\mathscr{M}_{\infty}^{*}$ correspond to transversal intersections of the perturbed stable/unstable manifolds is not proved in this paper.

To use the existing theory, for example the theory presented in [15] for the case of a periodically forced oscillator, we must verify that our system (23) can be recast in the appropriate form. Fortunately, this is very simple: rescale (23) by the energy to obtain the differential equations

$$
\begin{aligned}
& \dot{x}=\frac{\sin z}{\beta \cos x+\cos z}+\epsilon \frac{\cos y}{\beta \cos x+\cos z}, \\
& \dot{y}=1, \\
& \dot{z}=-\frac{\beta \sin x}{\beta \cos x+\cos z}+\epsilon \frac{\sin y}{\beta \cos x+\cos z} .
\end{aligned}
$$

Since (49) is nonsingular in an open neighborhood of the heteroclinic orbits, if we view $y$ as a time like variable, then it suffices to consider the equivalent periodically forced oscillator given by

$$
\begin{aligned}
& \dot{x}=\frac{\sin z}{\beta \cos x+\cos z}+\epsilon \frac{\cos t}{\beta \cos x+\cos z}, \\
& \dot{z}=-\frac{\beta \sin x}{\beta \cos x+\cos z}+\epsilon \frac{\sin t}{\beta \cos x+\cos z} .
\end{aligned}
$$

Using the energy relation on the heteroclinic orbits of (24), namely, the equation $\beta \cos x+\cos z=1-\beta$, we find the Melnikov function, as presented in [15], to be

$$
\frac{1}{1-\beta} \int_{-\infty}^{\infty} \sin z \sin t+\beta \sin x \cos t d t
$$

For initial points along an unperturbed heteroclinic orbit of (24), this function has the same zeros as the function defined by $\mathscr{M}_{\infty}^{*}$. This fact is easily seen by a computation similar to the derivation of $\mathscr{M}^{*}$, equation (37), from $\mathscr{M}$, equation (36).

As a consequence of these remarks and the standard theory of the Melnikov function for forced oscillators, we conclude that a simple zero of $\mathscr{M}_{\infty}^{*}$ corresponds to a transversal intersection of the perturbed stable/unstable manifolds. If there is such a zero, then by the Smale-Birkoff Theorem, cf. [12],[15], there is an invariant Cantor set on which our Poincare map for the ABC flow is topologically conjugate to a full shift on $k$ symbols for some integer $k \geq 2$. This is the chaotic invariant set we seek.

If $I_{s}^{2}+I_{c}^{2} \neq 0$, then $\theta \mapsto \mathscr{M}_{\infty}^{*}(\theta)$ has (infinitely many) simple zeros. We will determine when $I_{s}^{2}+I_{c}^{2} \neq 0$ by finding explicit expressions for $\cos x(t)$ and $\sin x(t)$. This is accomplished as before by integrating the unperturbed Hamiltonian system (24). 
Here, we have

$$
\tau=\int_{0}^{x(\tau)} \frac{1}{\sqrt{1-(1-\beta-\beta \cos \sigma)^{2}}} d \sigma .
$$

Equivalently,

$$
\tau \sqrt{\beta}=\int_{0}^{x(\tau)} \frac{1}{\sqrt{(1+\cos \sigma)(2-\beta(1+\cos \sigma))}} d \sigma .
$$

The form of the integrand suggests the substitution $u=1+\cos \sigma$. It transforms the last integral to

$$
\tau \sqrt{\beta}=-\int_{2}^{1+\cos x(\tau)} \frac{1}{u \sqrt{(2-u)(2-\beta u)}} d u .
$$

Then, using the indefinite integral

$$
\int \frac{1}{u \sqrt{(2-u)(2-\beta u)}} d u=-\frac{1}{2} \ln \left(\frac{4 \sqrt{(2-u)(2-\beta u)}-2(\beta+1) u+8}{u}\right),
$$

we find

$$
\cos x(\tau)=-\frac{(\beta-1) e^{4 \tau \sqrt{\beta}}+2(3-\beta) e^{2 \tau \sqrt{\beta}}+\beta-1}{(\beta-1) e^{4 \tau \sqrt{\beta}}-2(\beta+1) e^{2 \tau \sqrt{\beta}}+\beta-1}
$$

and

$$
\sin x(\tau)=4 \sqrt{1-\beta} \frac{e^{\tau \sqrt{\beta}}\left(e^{2 \tau \sqrt{\beta}}-1\right)}{(\beta-1) e^{4 \tau \sqrt{\beta}}-2(\beta+1) e^{2 \tau \sqrt{\beta}}+\beta-1} .
$$

Clearly $I_{s}$ and $I_{c}$ are convergent. Also, the integrand of $I_{c}$ is odd while the integrand of $I_{s}$ is even. It follows that $I_{c}=0$. We will evaluate the integral $I_{s}$ by a contour integration.

Define $w:=e^{z \sqrt{\beta}}$ and

$$
F(w):=4(1-\beta)^{-3 / 2} \frac{w\left(w^{2}-1\right)\left((1-\beta) w^{4}+2(\beta-3) w^{2}+1-\beta\right)}{\left(w^{4}+(2(1+\beta) /(1-\beta)) w^{2}+1\right)^{2}}
$$

to obtain

$$
I_{s}=\int_{-\infty}^{\infty} F(w) \sin ((1-\beta) z) d z
$$

Also, note that the poles of the integrand of $I_{s}$ correspond to the zeros of the denominator of $F$. To determine these zeros we factor this denominator as

$$
w^{4}+(2(1+\beta) /(1-\beta)) w^{2}+1=\left(w^{2}-u_{1}\right)\left(w^{2}-u_{2}\right)
$$

where

$$
u_{1}:=\frac{\sqrt{\beta}-1}{\sqrt{\beta}+1}, \quad u_{2}:=\frac{\sqrt{\beta}+1}{\sqrt{\beta}-1} .
$$

If $\mathbb{Z}$ denotes the set of integers, then the solutions of $e^{z \sqrt{\beta}}=u_{1}$ are

$$
\frac{1}{2 \sqrt{\beta}}\left(\ln \left(-u_{1}\right)+\pi i+2 k \pi i\right), \quad k \in \mathbb{Z},
$$


while the solutions corresponding to $u_{2}$ are

$$
\frac{1}{2 \sqrt{\beta}}\left(\ln \left(-u_{2}\right)-\pi i+2 k \pi i\right), \quad k \in \mathbb{Z} .
$$

The locations of the poles suggest integration around the rectangle $\Gamma$ in the complex plane whose vertices are $T, T+i \pi / \sqrt{\beta}, T-i \pi / \sqrt{\beta}$ and $-T$. In effect, for sufficiently large $T>0$, this contour encloses exactly two poles of the integrand, namely

$$
z_{1}:=\frac{1}{2 \sqrt{\beta}}\left(\ln \left(-u_{1}\right)+\pi i\right), \quad z_{2}:=\frac{1}{2 \sqrt{\beta}}\left(\ln \left(-u_{2}\right)+\pi i\right) .
$$

The function $F$ defined above is odd and, for all $w \neq 0$, we have $F(1 / w)=$ $-F(w)$. Using these facts and the identity $\sin z=\left(e^{i z}-e^{-i z}\right) /(2 i)$, we obtain

$$
I_{s}=-i \int_{-\infty}^{\infty} F\left(e^{z \sqrt{\beta}}\right) e^{i(1-\beta) z} d z
$$

For notational convenience, let $\mathscr{K}:=\mathscr{K}(\beta)=(1-\beta) \pi /(2 \sqrt{\beta})$. After an easy computation, we find the corresponding path integral along the upper edge of $\Gamma$ is just $e^{-2 \mathscr{K}}$ times the path integral along the lower edge. Also, by using the obvious estimates, it is easy to see that the path integrals along the vertical edges of $\Gamma$ approach zero as $T$ increases without bound. Thus, we have

$$
\begin{aligned}
I_{s} & =-i\left(1+e^{-2 \mathscr{K}}\right)^{-1} \int_{\Gamma} F\left(e^{z \sqrt{\beta}}\right) e^{i(1-\beta) z} d z \\
& =2 \pi\left(1+e^{-2 \mathscr{K}}\right)^{-1}\left(\operatorname{Res}\left(z_{1}\right)+\operatorname{Res}\left(z_{2}\right)\right) .
\end{aligned}
$$

To determine the residues, we will use the definitions

$$
F_{1}(w):=\left(w^{2}-u_{1}\right)^{2} F(w) \text { and } F_{2}(w):=\left(w^{2}-u_{2}\right)^{2} F(w)
$$

In fact, after computing the Laurent series of the integrand, we find

$$
\begin{aligned}
& \operatorname{Res}\left(z_{1}\right)=\frac{e^{i(1-\beta) z_{1}}}{4 \beta u_{1}^{2}}\left(\sqrt{\beta} e^{z_{1} \sqrt{\beta}} F_{1}^{\prime}\left(e^{z_{1} \sqrt{\beta}}\right)-(2 \sqrt{\beta}-i(1-\beta)) F_{1}\left(e^{z_{1} \sqrt{\beta}}\right)\right), \\
& \operatorname{Res}\left(z_{2}\right)=\frac{e^{i(1-\beta) z_{2}}}{4 \beta u_{2}^{2}}\left(\sqrt{\beta} e^{z_{2} \sqrt{\beta}} F_{2}^{\prime}\left(e^{z_{2} \sqrt{\beta}}\right)-(2 \sqrt{\beta}-i(1-\beta)) F_{2}\left(e^{z_{2} \sqrt{\beta}}\right)\right) .
\end{aligned}
$$

To simplify the expression for the sum of the residues, define

$$
\mathscr{A}:=\cos \left(\frac{1-\beta}{2 \sqrt{\beta}} \ln \left(-u_{2}\right)\right), \quad \mathscr{B}:=\sin \left(\frac{1-\beta}{2 \sqrt{\beta}} \ln \left(-u_{2}\right)\right)
$$

and compute

$$
e^{i(1-\beta) z_{1}}=e^{-\mathscr{K}}(\mathscr{A}-\mathscr{B} i), \quad e^{i(1-\beta) z_{2}}=e^{-\mathscr{H}}(\mathscr{A}+\mathscr{B} i) .
$$

Also, since $u_{1}=1 / u_{2}$, note that 


$$
e^{z_{1} \sqrt{\beta}} e^{z_{2} \sqrt{\beta}}=-1 .
$$

Thus, we can express $\operatorname{Res}\left(z_{1}\right)$ in terms of the components of $\operatorname{Res}\left(z_{2}\right)$.

For this, note that $F_{1}$ is an odd function, $F_{1}^{\prime}$ is an even function, and compute

$$
\begin{aligned}
& F_{1}(1 / w)=-\frac{1}{w^{4} u_{2}^{2}} F_{2}(w), \\
& F_{1}^{\prime}(1 / w)=\frac{1}{w^{2} u_{2}^{2}} F_{2}^{\prime}(w)-\frac{4}{w^{3} u_{2}^{2}} F_{2}(w)
\end{aligned}
$$

Again, for notational convenience, define $\mathscr{L}:=\sqrt{-u_{2}}$. Then, after a computation, the residues may be expressed as follows:

$$
\begin{aligned}
& \operatorname{Res}\left(z_{1}\right)=\frac{1}{4 \beta u_{2}^{2}} e^{-\mathscr{K}}\left((\mathscr{A}-\mathscr{B} i)\left(-i \mathscr{L} \sqrt{\beta} F_{2}^{\prime}(i \mathscr{L})+(2 \sqrt{\beta}-i(1-\beta)) F_{2}(i \mathscr{L})\right),\right. \\
& \operatorname{Res}\left(z_{2}\right)=\frac{1}{4 \beta u_{2}^{2}} e^{-\mathscr{H}}\left((\mathscr{A}+\mathscr{B} i)\left(i \mathscr{L} \sqrt{\beta} F_{2}^{\prime}(i \mathscr{L})-(2 \sqrt{\beta}-i(1-\beta)) F_{2}(i \mathscr{L})\right) .\right.
\end{aligned}
$$

Note that $F_{2}^{\prime}(i \mathscr{L})$ is real and define the real number $F_{2}^{*}:=-i F_{2}(i \mathscr{L})$. Using the formula for $I_{s}$ given by equation (50), an easy calculation yields

$$
\begin{aligned}
I_{s} & =\frac{\pi e^{-\mathscr{H}}}{\beta u_{2}^{2}\left(1+e^{-2 \mathscr{H}}\right)}\left(\mathscr{B} \sqrt{\beta}\left(2 F_{2}^{*}-\mathscr{L} F_{2}^{\prime}(i \mathscr{L})\right)-\mathscr{A}(1-\beta) F_{2}^{*}\right) \\
& =\frac{\pi e^{-\mathscr{H}}}{\beta u_{2}^{2}\left(1+e^{-2 \mathscr{H}}\right)} F_{2}^{*}(\mathscr{B}(\beta+4 \sqrt{\beta}-1)-\mathscr{A}(1-\beta)) .
\end{aligned}
$$

If $0<\beta<1$, then $F^{*} \neq 0$. Thus, $I_{s}=0$ if and only if

$$
\tan \left(\frac{1-\beta}{2 \sqrt{\beta}} \ln \left(\frac{1+\sqrt{\beta}}{1-\sqrt{\beta}}\right)\right)=\frac{1-\beta}{\beta+4 \sqrt{\beta}-1} .
$$

In fact, the last equation has exactly one root for $\beta$ in the specified range, $\beta \approx 0.1466$. Thus, except at this one point, our first order computation shows the perturbed stable and unstable manifolds intersect transversally and, using the Smale-Birkoff Theorem, the corresponding perturbed flow is chaotic in a zone near these manifolds. Perhaps a second order computation would show the same is true at the exceptional value of $\beta$.

The results of some numerical experiments on the system (23) are depicted in Figures 1-3. A "dot" represents a point on an orbit of the Poincaré map defined above for the system (23). The Poincare section is a portion of the $x z$ plane.

Using the formulas given above, the position of the unperturbed resonant tori can be computed explicitly. For example, the system (23) with $\beta=0.16$ and $\epsilon=0.01$ has a $(3: 1)$ resonant torus. The corresponding unperturbed resonant orbit of $(24)$ crosses the $x$ axis at the point $(x, z) \approx(1.542,0.0)$. Figure 1 illustrates the persistence of six periodic points near this $(3: 1)$ resonance, as predicted by the theoretical results.

Figure 3 and Figure 4 suggest some of the structure in the stochastic layer that forms after breaking the heteroclinic orbits of the unperturbed Poincare map. As predicted, the orbit structure appears to be very complex. 


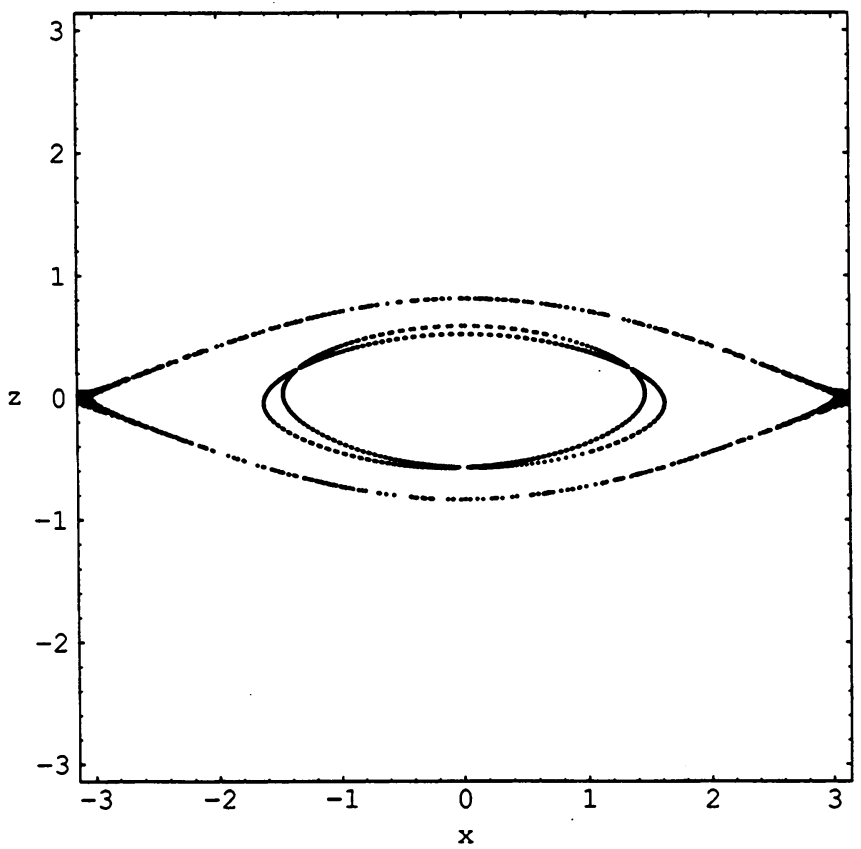

FIGURe 2. Orbits of the Poincare map for (23), with $\beta=0.16$ and $\epsilon=0.01$, near the $(3: 1)$ resonance and near the stochastic layer.

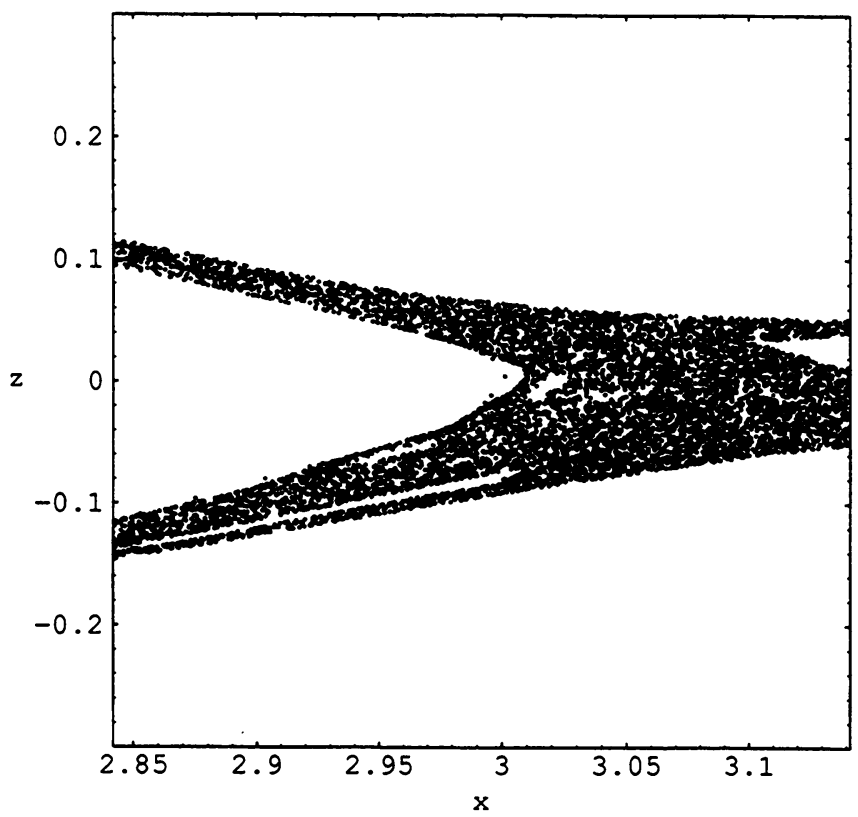

FIGURE 3. Blow-up of Figure 2 depicting 42,317 iterates of the point $(x, z)=(3.14,0.0)$. 


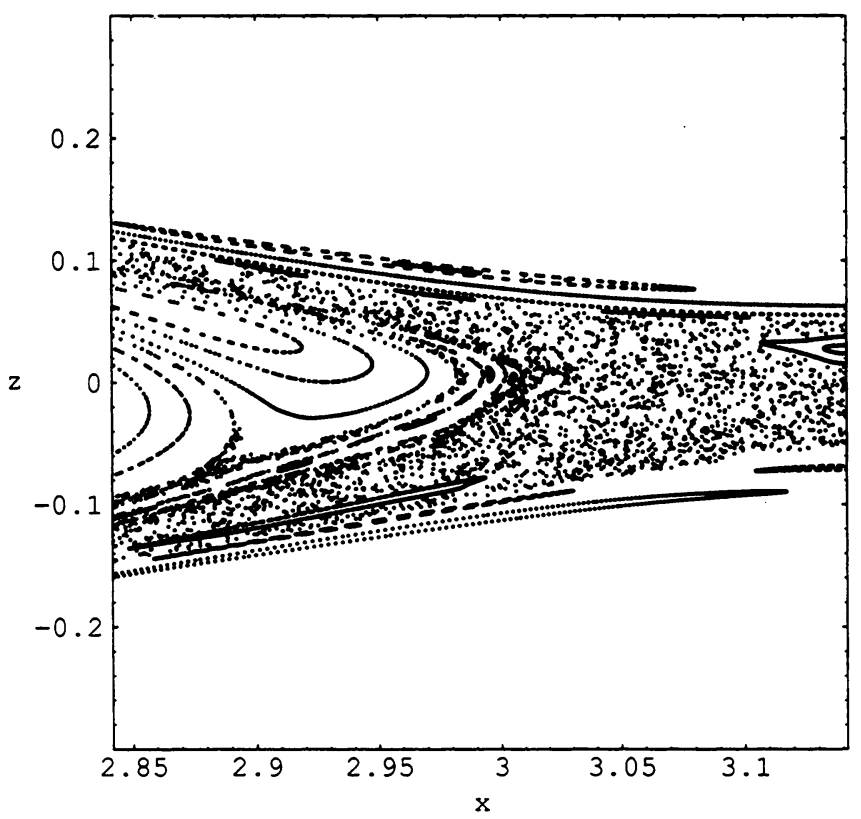

FIGURE 4. Blow-up of Figure 2 depicting several orbits near the "boundary" of the orbit plotted in Figure 3.

\section{REFERENCES}

1. V. I. Arnold, Mathematical methods of classical mechanics, Springer-Verlag, New York, 1978.

2. Geometric methods in the theory of ordinary differential equations, Springer-Verlag, New York, 1982.

3. P. F. Byrd and M. D. Friedman, Handbook of elliptic integrals for engineers and scientists, 2nd ed., Springer-Verlag, Berlin, 1971.

4. C. Chicone, The topology of stationary curl parallel solutions of Euler's equations, Israel J. Math. 39 (1981), 161-166.

5. Bifurcation of nonlinear oscillations and frequency entrainment near resonance, SIAM J. Math. Anal. 23 (1992), 1577-1608.

6. Lyapunov-Schmidt reduction and Melnikov integrals for bifurcation of periodic solutions in coupled oscillators, J. Differential Equations 112 (1994), 407-447.

7. Analysis (to appear).

8. S. P. Diliberto, On systems of ordinary differential equations, Contributions to the Theory of Nonlinear Oscillations, Ann. of Math. Stud., no. 20, Princeton Univ. Press, Princeton, NJ, 1950.

9. S. Friedlander, A. Gilbert and M. Vishik, Hydrodynamic instability and certain ABC flows, Preprint 1993.

10. S. Friedlander and M. Vishik, Instability criteria for the flow of an inviscid incompressible fluid, Phys. Rev. Let. 66 (1991), 2204-2206.

11. _ Instability criteria for steady flows of a perfect fluid, Chaos 2 (1992).

12. J. Guckenheimer and P. Holmes, Nonlinear oscillations, dynamical systems, and bifurcations of vector fields, 2nd ed., Springer-Verlag, New York, 1986.

13. D. Husemoller, Fibre bundles, McGraw-Hill, New York, 1966. 
14. V. K. Melnikov, On the stability of the center for time periodic perturbations, Trans. Moscow Math. Soc. 12 (1963), 1-57.

15. S. W. Wiggins, Introduction to applied nonlinear dynamical systems and chaos, SpringerVerlag, New York, 1990.

16. E. T. Whittaker and G. N. Watson, $A$ course of modern analysis, Macmillan, New York, 1946.

Department of Mathematics, University of Missouri, Columbia, Missouri 65211

E-mail address: carmen৫chicone.cs.missouri.edu 